\title{
Population differentiation of polygenic score predictions under stabilizing selection
}

\author{
Sivan Yair $^{1}$ and Graham Coop ${ }^{1}$ \\ ${ }^{1}$ Center for Population Biology and Department of Evolution and Ecology, University of California, Davis
} To whom correspondence should be addressed: ssyair@ucdavis.edu

\section{Abstract}

Given the many loci uncovered by genome-wide association studies (GWAS), polygenic scores have become central to the drive for genomic medicine and have spread into various areas including evolutionary studies of adaptation. While promising, these scores are fraught with issues of portability across populations, due to the mis-estimation of effect sizes and missing causal loci across populations not represented in large-scale GWAS. The poor portability of polygenic scores at first seems at odds with the view that much of common genetic variation is shared among populations (Lewontin, 1972). Here we investigate one potential cause of this discrepancy: phenotypic stabilizing selection drives the turnover of genetic variation shared between populations at causal loci. Somewhat counter-intuitively, while stabilizing selection to the same optimum phenotype leads to lower phenotypic differentiation among populations, it increases genetic differentiation at GWAS loci and reduces the portability of polygenic scores constructed for unrepresented populations. We also find that stabilizing selection can lead to potentially misleading signals of the differentiation of average polygenic scores among populations. We extend our baseline model to investigate the impact of pleiotropy, gene-by-environment interactions, and directional selection on polygenic score predictions. Our work emphasizes stabilizing selection as a null evolutionary model to understand patterns of allele frequency differentiation and its impact on polygenic score portability and differentiation.

\section{Introduction}

Lewontin's foundational early survey of human genetic variation found that much of genetic variation is found within human populations rather than between human populations, i.e. that human populations are only weakly differentiated at the level of individual loci and most common variation is shared among populations (Lewontin, 1972). Thus it provided a strong refutation of an alternative view in which genetic variation would be partitioned among mostly invariable populations and has become a classic work in discrediting the view of discrete human races. Lewontin's finding is reflected by low estimates of $F_{S T}$ among populations ( $\mathrm{Li}$ et al., 2008), i.e. only a small proportion $(\sim 10 \%)$ of the total allelic variance is attributable to differences in frequency between populations. Lewontin's early work made use of breakthroughs in the measurement of protein variation using electrophoretic mobility and earlier surveys of blood groups. Since then, the findings of low $F_{S T}$ have been found to hold over many different markers across the genome (Barbujani et al., 1997; Jorde et al., 2000; Rosenberg et al., 2002; Conrad et al., 2006; Li et al., 2008; Bergström et al., 2020). While some selected loci that are highly differentiated among human populations have been uncovered (e.g. underlying loci in skin pigmentation and infectious disease immunity; see Fan et al., 2016, for a review), there are relatively few such strongly selected loci in the genome (Coop et al., 2009; Hernandez et al., 2011).

Lewontin's results also have important implications for our a priori expectations for the partitioning of phenotypic variation within and among human populations. That is because for phenotypes evolving neutrally we expect the proportion of additive genetic phenotypic variance attributable to among populations differences to be $\approx 2 F_{S T}$ when all individuals are measured in a common set of environments (Wright, 1951; Lande, 1976; Rogers and Harpending, 1983; Lande, 1992; Whitlock, 1999; Edge and Rosenberg, 2015). Thus in humans, we expect only $\sim 18 \%$ of human neutral phenotypic variation to be due to between population 
differences when measured in a common environment. Phenotypes subject to divergent selection among populations can be over-dispersed compared to this null, while phenotypes subject to stabilizing selection with the same selective optimum are expected to be less differentiated among populations. To distinguish between genetic and environmental contributors of differences among populations, researchers often turn to settings like common gardens in an effort to eliminate environmental differences. However, it is obviously not feasible to measure human phenotypes in a common environment (a major drawback of studies that investigated population level phenotypic variation; Relethford and Lees, 1982; Chakraborty, 1990). Thus for the vast majority of complex traits we do not know the role of genetics, let alone natural selection in phenotypic differences among human populations.

These questions have received renewed interest in human genetics due to genome-wide association studies (GWAS), which have found common variation associated with many phenotypes within populations. Indeed how generalizable variants associated with disease are across populations (portability) is a key concern as GWAS have strong biases towards individuals of European ancestry and studies of other populations are much smaller (Li and Keating, 2014; Popejoy and Fullerton, 2016; Macarthur et al., 2017; Martin et al., 2019). GWAS have revealed that most phenotypes are highly polygenic within populations (Loh et al., 2015; Shi et al., 2016; Boyle et al., 2017) and that much of the genetic variance is additive (reviewed in Hill et al., 2008). These observations have led to renewed interest in human genetics in estimating additive genetic values, the additive contribution of polymorphisms to phenotypic differences among individuals. One common approach to predict an individual's additive genetic trait value is based on a polygenic score, the sum of an individual's genotype at trait-associated loci weighted by their estimated effects. Predictions based on polygenic scores are being explored in a number of clinical settings and more generally as a tool for understanding the genetic basis of disease and phenotypic variability.

Currently, polygenic scores are often poor predictors of the additive genetic value underlying traits, and therefore phenotypes, because they aggregate across many loci with slightly mis-estimated effects and they only account for a subset of loci that contribute to trait variation (Mostafavi et al., 2020; Sakaue et al., 2020). Moreover, the associated loci are usually not the causal loci underlying trait variation, instead they tag the effects of linked causal sites. The interpretation of the effect size of an associated variant can be tricky because of: (i) linkage disequilibrium (LD), whereby it absorbs the effects at correlated causal sites (Vilhjálmsson et al., 2015; Horikoshi et al., 2017; Lam et al., 2019; Wang et al., 2020; Weissbrod et al., 2021); (ii) population stratification, whereby it absorbs the effects of covarying environments (Haworth et al., 2019; Kerminen et al., 2019; Sakaue et al., 2020; Trochet and Hussin, 2020; Isshiki et al., 2021); and (iii) gene-by-environment $(\mathrm{GxE})$ or gene-by-gene $(\mathrm{GxG})$ interactions, whereby its estimate is averaged over the interacting environmental contexts or genetic backgrounds in the sample (Brown et al., 2016; Horikoshi et al., 2017; Coram et al., 2017; Adhikari et al., 2019; Bentley et al., 2019; Galinsky et al., 2019; Grinde et al., 2019; Veturi et al., 2019; Wojcik et al., 2019; Mathieson, 2021). As all of these factors can and will vary across populations, the effect sizes of alleles will differ across populations and so polygenic scores will have lower prediction accuracy, i.e. imperfect portability, across populations. There is wide agreement that these portability issues will have to be addressed if the future clinical use of polygenic scores is not to just further compound inequalities in healthcare ( $\mathrm{Li}$ and Keating, 2014; Popejoy and Fullerton, 2016; Martin et al., 2019).

In addition to individual level prediction, polygenic scores may help assess the contribution of selection to the genetic basis of phenotypic differentiation among human populations, in the absence of a way to evaluate phenotypic differences in a common garden setting. The variance of polygenic scores among populations should be proportional to $F_{S T}$ if the trait is neutrally evolving (Berg and Coop, 2014). An early application of this idea found polygenic signals of selection on height within Europe (Turchin et al., 2012; Berg and Coop, 2014), where polygenic scores were over-differentiated among populations compared to this neutral prediction. Importantly the null distribution of this test of neutrality at the level of the phenotype does not rely on the polygenic scores being accurate and so changes in $\mathrm{LD}, \mathrm{GxG}$, and GxE should not cause false signals. However, the results are very sensitive to slight biases in estimated effect sizes due to population structure, and indeed the signal of polygenic selection on height turned out to be almost entirely due to stratification (Berg et al., 2019; Sohail et al., 2019; Refoyo-Martínez et al., 2020). More generally the imperfect portability of polygenic scores, missing genetic variation, and the fact that the mean environmental contribution to phenotypes can vary greatly between populations, raises significant concerns about overinterpreting differences in mean polygenic scores as genetic differences in the average phenotype among 
populations (Berg and Coop, 2014; Novembre and Barton, 2018; Coop, 2019; Rosenberg et al., 2019; Harpak and Przeworski, 2021).

Even with perfectly estimated effects at the causal loci with significant trait associations, the prediction accuracy of polygenic scores will be limited because GWAS only identify loci with common alleles that contribute enough variance to exceed some significance threshold determined by the sample size. Therefore, an allele that is rare in the GWAS sample but common elsewhere will not be discovered, leading to a greater reduction in the variance accounted for, or prediction accuracy, in non-represented populations. (Liu et al., 2015; Martin et al., 2017a,b; Curtis, 2018; Kim et al., 2018; Bentley et al., 2019; Wojcik et al., 2019; Wang et al., 2020; Conti et al., 2021; Cavazos and Witte, 2021). The impact of genetic differentiation on prediction accuracy in non-represented populations can be large, as it has been found that many variants contributing to trait variation in European GWAS samples were not at a high enough frequency to be detected in other populations, suggesting different alleles contribute greatly to the trait variance in each population (Liu et al., 2015; Durvasula and Lohmueller, 2021). This may appear to contradict Lewontin's observation of minor allele frequency differences between populations. However, allele frequency differentiation for complex traits under selection can occur at a rate faster than drift (Durvasula and Lohmueller, 2021). This would lead to noisy estimates of additive genetic values, and so reduced prediction accuracy of polygenic scores.

Here we describe how stabilizing selection on complex traits, a sensible null model for their evolution, leads to inaccurate genetic trait predictions for individuals more distantly related to those included in a GWAS sample, despite overall low genetic differentiation and no genetically-based trait differentiation among populations. Under stabilizing selection, intermediate trait values have the highest fitness, with decreasing fitness with distance from that optimum. Many quantitative traits have been shown to experience stabilizing selection in humans (e.g. Sanjak et al., 2018) as well as across many other species (e.g. Bumpus, 1899; Kingsolver et al., 2001; de Villemereuil et al., 2020). Stabilizing selection also contributes to the lack of variation in morphological traits within and between closely related species and the morphological constancy of traits in the fossil record (Gingerich, 1983; Weber, 1990; Barton and Keightley, 2002; Hill and Kirkpatrick, 2010; Houle et al., 2017). Under parameter ranges estimated from empirical studies, we combine simulations of stabilizing selection on complex traits with analytical models of its effect on genetic differentiation to investigate the impact of stabilizing selection on the prediction accuracy of polygenic scores and the chance of false signals of directional polygenic adaptation.

We focus initially on scenarios in which the trait optimum is always the same among populations, leading to levels of trait differentiation among populations much smaller than neutrality. We study differentiation between a pair of populations, either an ancestral and descendant population or a pair of contemporary populations, in which the results of a GWAS in one of those populations is used to make trait predictions in the other. In doing so we provide a polygenic score perspective on earlier investigations into the relationship between population structure, stabilizing selection, and quantitative trait variation (e.g. Narain and Chakraborty, 1987; Lande, 1991; Goldstein and Holsinger, 1992; Latta, 1998; Le Corre and Kremer, 2003). In our baseline scenario, stabilizing selection occurs on a single additive trait where alleles have the same effect in different populations (reflecting no GxG or GxE interactions). We explored three variations of this base scenario: (i) gene-by-environment interactions causing an imperfect correlation in allelic effect sizes between populations, (ii) pleiotropy, whereby a mutation can influence more than one trait under selection, and (iii) the same change in the optimal phenotype after the pair of populations diverged from a common ancestor. We show how these factors affect the reduced prediction accuracy of polygenic scores and can readily lead to patterns of polygenic score differentiation rife with the potential for misinterpretation.

\section{Model Background}

An individual's additive genetic value $G_{i}$ is the sum of the additive effects of all alleles they carry,

$$
G_{i}=\sum_{l} a_{l} g_{i l},
$$

where $a_{l}$ represents the additive effect of an allele relative to another at locus $l$ and $g_{l}$ is the number of copies of that allele carried by the individual. All of these loci denoted by $l$ are polymorphic within a specified population from which the individual was drawn. Note that the additive genetic value does not represent 
an absolute measure of an individual's phenotype, and instead represents the additive contribution of the polymorphisms they carry to their deviation from their population's mean.

Under a constant selective environment, stabilizing selection keeps the population mean phenotype close to the optimum and decreases the phenotypic variance in the population because individuals on both tails of the distribution have lower fitness (Lande, 1976; Lande and Arnold, 1983). To understand the process by which stabilizing selection reduces the phenotypic variance, we focus on the additive genetic variance for a set of unlinked loci,

$$
V_{A}=\operatorname{Var}(G)=\underbrace{\sum_{l} a_{l}^{2} \operatorname{Var}\left(g_{l}\right)}_{\text {genic variance }}+\underbrace{\sum_{l \neq l^{\prime}} a_{l} a_{l^{\prime}} \operatorname{Cov}\left(g_{l}, g_{l^{\prime}}\right)}_{\text {LD contribution }},
$$

in which the first component refers to the additive genic variance $\left(V_{a}\right)$ and the second component accounts for the contribution of linkage disequilibrium (LD) among loci that contribute to the variance. In the short term, stabilizing selection reduces the phenotypic variance by generating negative LD between like-effect alleles, thereby limiting extreme phenotypes, which is known as the Bulmer effect (Bulmer, 1971). The additive genetic variance quickly reaches an equilibrium reflecting a balance between selection producing negative LD and recombination and chromosome segregation breaking up that generated LD. If the genetic basis of trait variation was truly infinitesimal, i.e. made up of loci of infinitely small effect, there would be no further reduction in the variance due to selection. Thus only genetic drift would erode variation in the long term, because it reduces heterozygosity $\left(\operatorname{Var}\left(g_{l}\right)\right)$. However, while the loci we discover with GWAS contribute very small effects, these effects are not infinitesimally small and so they can be directly acted on by selection (Simons et al., 2018; Sella and Barton, 2019).

The long-term genetic response to stabilizing selection is driven by changes in the additive genic variance, in particular the variance in genotypes at a locus. The full analysis of such models is challenging as changes in allele frequencies and covariances must be tracked over many loci (e.g. Turelli and Barton, 1990). Under moderate strengths of stabilizing selection and a constant environment, the population mean phenotype stays very close to the optimum through rapid, small fluctuations at many individual loci. Then since the genotypic variance at a locus is proportional to the expected heterozygosity, stabilizing selection reduces the additive genic variance by reducing heterozygosity. Selection at the individual loci underlying variation in the trait is in many cases well approximated by underdominant selection, in which the more common allele fixes and the minor allele is lost (Figure 1A; Wright, 1935; Robertson, 1956). Due to underdominant selection at the locus level, stabilizing selection removes polymorphisms at a faster rate than neutrality with a selection coefficient proportional to their squared effect sizes (see Simons et al., 2018; Sella and Barton, 2019; Hayward and Sella, 2021, for recent applications of such models to understand GWAS variation within populations).

To understand the long-term turnover in the genetic basis of trait variation, from here on we focus on the additive genic variance, $V_{a}$. When the population is at mutation-drift-selection equilibrium, the phenotypic variance is constant, with mutations replenishing variation removed by stabilizing selection and genetic drift. We begin by thinking of an ancestral population at equilibrium and the loss of ancestral phenotypic variance over time, where as time goes on less and less of the variance in the descendant population will be contributed by ancestral polymorphisms. We assume a model of Gaussian stabilizing selection where the width of the fitness peak is determined by $w\left(w^{2}\right.$ is equivalent to $V_{S}$ in other models of stabilizing selection), which will approximate any symmetric quadratic stabilizing selection model when the mean trait is close to its optimum. We also assume a normal distribution of mutant effects and thousands of unlinked loci contributing to trait variation; see Appendix A.1 for simulation details. The reduction in variance contributed by a particular polymorphism is the same as the reduction in heterozygosity at that site. We can use a common approximation for the per generation loss of heterozygosity (e.g. Keightley and Hill, 1988) to predict the reduction in heterozygosity, or additive genic variance $\left(V_{a}\right)$, contributed by ancestral variants (anc) in the descendant population (desc) after $t$ generations for alleles with effect size $a$,

$$
\frac{V_{a[\text { anc, desc }]}(a, t)}{V_{a}(a, 0)}=(1-1 / 2 N)^{t} \times\left(1-a^{2} / 4\left(w^{2}+V_{P}\right)\right)^{t},
$$

where $V_{P}$ is the total population trait variance. On the right-hand side, the first bracketed term is the per generation reduction due to drift and the second term is the reduction due to stabilizing selection. Selection 


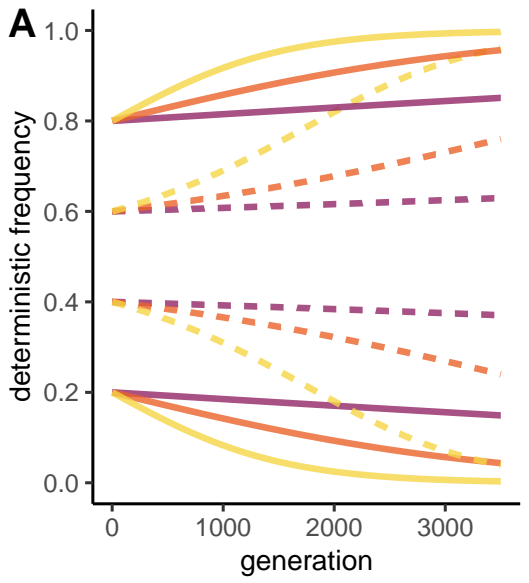

boundary distance $\quad$ effect size

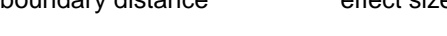

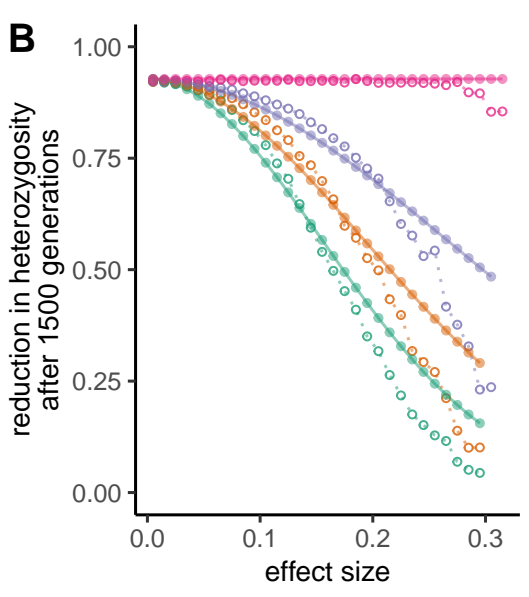

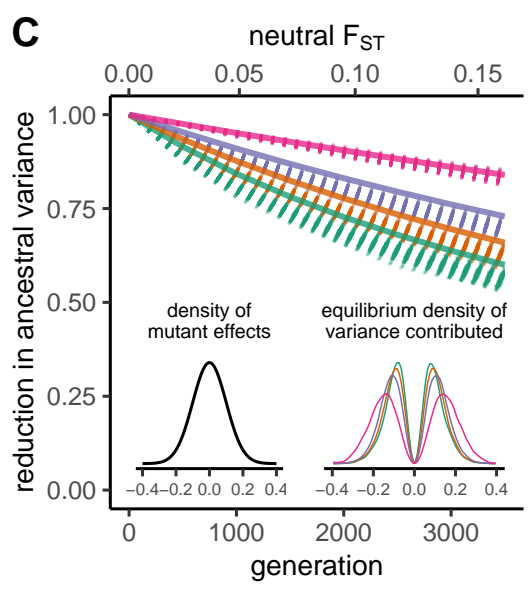

width of fitness peak 0.3 $\rightarrow \mathrm{w}=4 \rightarrow \mathrm{w}=5 \rightarrow \mathrm{w}=7 \rightarrow$ neutral

Figure 1: A) Deterministic allele frequency trajectories, assuming an infinite population size, based on the underdominant model, at a locus that contributes to the variance of a trait under stabilizing selection (in this case $w=4$ ). The trajectory differs according to effect size and starting frequency. Note the symmetry for starting frequencies that are the same distance to their closest boundaries. B) Reduction in heterozygosity at loci that contributed to the variance 1500 generations ago in a population with $N_{e}=10,000$. Each open point represents the midpoint of the effect size bin of width 0.01 , within which we averaged heterozygosity from 100 simulations. Each filled point represents our analytical predictions for that midpoint. C) Reduction over time in the total variance contributed by polymorphisms in an ancestral population. Dashed lines show results from simulations and solid lines show analytical predictions. The axis at the top shows the expected neutral $F_{S T}$ between the ancestral and descendant population for the time scales of divergence shown on the bottom axis. The inset on the left shows the distribution from which mutant effect sizes are drawn. For the results shown we used a normal distribution with standard deviation of 0.1 . The inset on the right shows the equilibrium density of variance contributed from each effect size. As the width of the fitness peak increases, and thus stabilizing selection weakens, mutations with large effects can drift to higher frequencies and contribute a greater proportion of the trait's variance.

on an allele is stronger when the fitness peak is narrower (i.e. when $w$ is smaller) and when the allele's effect is larger, causing a greater reduction in heterozygosity at those sites (Figure 1B). The reduction in heterozygosity corresponds to an elevation in $F_{S T}$ between the ancestral and descendant populations. We can average the reduction in heterozygosity across all sites, weighting by the distribution of effect sizes and genic variance contributed by a given effect size, to predict the total reduction in $V_{a}$ over time (Figure 1C). In the example shown in Figure 1, this total reduction tends to be weaker than what we see for the largest effect polymorphisms, because most sites that contribute to the variance are of small effect. This is because we assumed (i) most mutations have small effects, and (ii) due to stabilizing selection, at equilibrium the distribution of observed effects at variance-contributing sites is more narrow than the distribution of mutant effects. Thus when the variance of the mutant effect size distribution is $1 / 4$ of that used in Figure $1 \mathrm{C}$, we see less of a reduction in the variance contributed by ancestral polymorphisms (Figure S1). In Appendix A.3 we develop a different approximation to the decay in variance that performs somewhat better (Figure S2).

\section{Accuracy of polygenic score predictions}

To understand the effect of stabilizing selection and drift on the prediction accuracy of polygenic scores in isolation from other sources of bias, we make the simplifying assumption that GWAS identify associations between polymorphisms and trait variation only at causal loci. For a GWAS within a population to identify a locus as being associated with the trait, the locus has to be polymorphic in that population and its 
phenotypic association has to achieve some level of statistical significance (i.e. contribute above some level of variance to the trait). For those causal loci with significant associations, we also assume their effects are estimated perfectly and for the moment that these true effects do not vary within the sample, population the sample was drawn from, or across populations (i.e. populations experience the same set of environments). We also assume their effects are strictly additive. At the trait-associated loci, one can sum the additive effects of all alleles an individual carries at a predefined set of markers to form a polygenic score.

We are interested in the reduction in prediction accuracy for a population not represented in the GWAS sample, relative to the prediction accuracy of those represented populations. To explore this we consider the genetic differentiation between a pair of populations, $\mathrm{A}$ and $\mathrm{B}$, in which the GWAS sample is drawn from population A but not population B. We assume for simplicity that A and B split from a common ancestral population without subsequent gene flow. When allelic effects do not vary between populations, we can quantify the reduction in phenotype prediction accuracy $\left(r^{2}\right)$ of polygenic scores, compared to using additive genetic values, constructed for a population as

$$
r^{2}=\frac{\operatorname{Var}(P)}{\operatorname{Var}(G)},
$$

where $P_{i}$ is an individual's polygenic score and $G_{i}$ is their additive genetic value. Since an individual's polygenic score is only part of their additive genetic value when a GWAS estimates true effects, this reduction can be understood as the proportion of the total additive genetic variance, or proportion of the heritability, explained by GWAS-significant sites (Appendix A.2). The ratio of $r^{2}$ in population B to population A quantifies the reduction in prediction accuracy due to a lack of a GWAS for population B. Note that our definition of the prediction accuracy in population B is the squared correlation of the deviation of an individual's polygenic score with its deviation in phenotype, where both of these deviations are with respect to the mean phenotype in population B. This definition matches typical polygenic score practices where predictions are statements about the departure of an individual from their ancestry group's mean genetic value, rather than a prediction of their departure from the mean genetic value of the GWAS population. However, this definition of prediction accuracy does not include discrepancies from the evolution of mean polygenic scores and phenotypes among populations, meaning it does not account for a systematic shift in polygenic scores between populations. We turn to this point in the next section.

We begin with the simplified case in which all causal loci that are polymorphic in population A have been identified, such that polygenic scores perfectly predict additive genetic values in population A. In Figure 2A, we use simulations and analytical predictions, to show how prediction accuracy in population B decreases with increasing time since its common ancestor with population A. These time depths reflect a scale of genetic differentiation along which various populations could fall. We see slightly weaker reductions in prediction accuracy with time when the variance of the mutant effect size distribution is quartered (Figure S4). The demographic and evolutionary histories of both populations are the same, such that their population means are both extremely close to the optimum and their trait variances are very similar. Since in this simplified case, all variance-contributing loci were ascertained in population $\mathrm{A}$, the only reason the full genic variance in population B was not captured is because private polymorphisms contribute to the variance in each population. At the time of divergence between the pair of populations, they entirely share their genetic basis of trait variation. Then as stabilizing selection and drift remove polymorphisms (at equilibrium), new mutations replenish them at different sites in each population, leading to the same total variance but different genetic bases of that variance (assuming a very large mutational target). Without gene flow between the pair of populations, the polymorphisms that arose since their common ancestor will remain private to each population and will thus not contribute to the variance in the other population. Therefore the polymorphisms in population A that also contribute to the variance in population B must be ancestrally shared polymorphisms, specifically at those loci in which ancestral polymorphisms were not removed by drift or selection in either descendant population. Note that these polymorphisms will on average contribute less variance and have a higher chance of contributing different variances in each descendant population with time since the common ancestor of the populations.

As polygenic scores from ancient DNA are being used to investigate the phenotypic diversity of ancient human populations (Irving-Pease et al., 2021), we also considered the prediction accuracy in a population ancestral to population A of a polygenic score constructed using variants found in modern population A. The proportion of variance in the ancestral population explained by polymorphisms in population A on average 

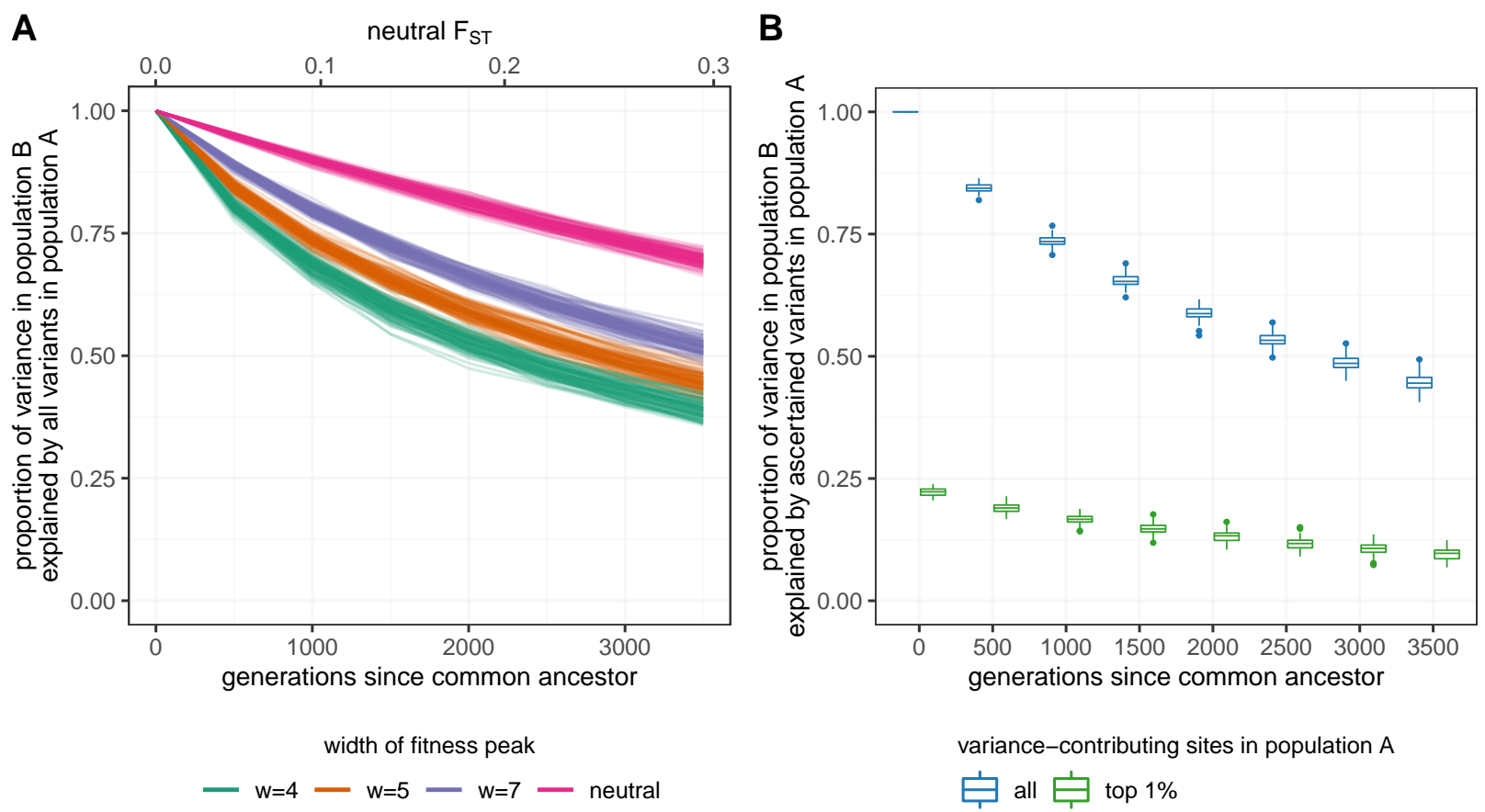

Figure 2: A) Reduction of prediction accuracy in population B, when all variance-contributing polymorphisms in population A were ascertained, with increasing time since the common ancestor of populations A and B. Lines show results from simulations, in which we recorded results every 500 generations. B) Distribution of the reduction in prediction accuracy for population B from simulations with $w=5$, either when all variance-contributing polymorphisms in population A were ascertained, or when the top $1 \%$ of variance-contributing polymorphisms in population A were ascertained.

equals the proportion of variance in population A explained by polymorphisms in the ancestral population (see Appendix A.3.2 for an explanation). Thus in expectation, the reduction in polygenic prediction accuracy for this ancestral population is equivalent to the reduction in variance contributed by that ancestral population to the present (see Figure 1C and Figure S5). While ancient individuals were likely not drawn from populations directly ancestral to present-day populations, we should observe the same general patterns with genetic differentiation between the ancient and present-day population.

To more realistically explore the impact of GWAS ascertainment, we focus on a case in which a GWAS uncovered the top $1 \%$ of variance-contributing polymorphisms in population A. Under our choice of effect size distribution and strengths of stabilizing selection, these polymorphisms explain just under $25 \%$ of the additive genic variance in population A. Unsurprisingly there is also a substantial drop in the variance explained in population B compared to the case in which all polymorphisms in population A are ascertained (Figure 2B). These patterns hold under directional selection for the optimum shifts we considered (up to two standard deviations of the trait distribution; Figure S6A). When only the top 1\% in population A are discovered by GWAS, only those shared polymorphisms that contribute relatively high variance in population A can contribute to the variance explained in population B. Thus while ancestrally shared polymorphisms are expected to contribute the same variance in each descendant population, on average those in the top $1 \%$ in population A contribute slightly higher variance in population A, because of their one-sided ascertainment (Figure S3A). However, this effect seems to be rather small and the relative loss of prediction accuracy in population B with the divergence of the two populations is similar to the case when all polymorphisms in A are used (Figure S3B,C).

Gene by environment interactions (GxE) combined with environmental differences between the GWAS sample and unrepresented population result in lower prediction accuracies in the unrepresented population, relative to the baseline scenario we just discussed (Figure S6). We assume the environments of the descendant 
populations change at their time of divergence from the ancestral population, and that these environmental changes do not generate a difference in the mean environment nor the optimum phenotype. To model GxE we perturb effect sizes in both descendant populations away from ancestral effect sizes by a small normal deviate, such that effect sizes have the same correlation between all three pairs of populations (either 0.9 or 0.95 , depending on the simulation). Two factors contribute to why we find lower prediction accuracies under GxE. First, the effect sizes estimated by the GWAS in population A are only partially correlated with the true effects in population B. Thus, noise is introduced to the predictions when using the effect sizes from A for polygenic prediction in B. In Appendix A.2.1 we describe how we modify the equation for the reduction in prediction accuracy when the effect sizes between the GWAS sample and the prediction population are only partially correlated. Second, the slight changes in effect sizes, due to environmental changes experienced by each descendant population after they shared a common ancestor, transiently inflate the variance in a population relative to equilibrium conditions, leading to a faster removal of ancestral polymorphisms and thus fewer shared polymorphisms between the population pair. Specifically, at equilibrium, most common alleles have small effects. After an environmental change, many of these previously near-zero effect alleles tend to shift toward larger effect sizes (Figure S7). The accumulation of these minor shifts at many sites leads to an increase in the total variance within the descendant populations, and these changes are not correlated between them, so prediction accuracy is reduced.

We extend our results to a model of pleiotropy, in which mutations influence multiple traits under stabilizing selection. In our implementation of pleiotropy in simulations, a mutation's effect on each trait is uncorrelated and we standardized our simulations such that each strength of stabilizing selection on the trait would correspond to the same average selection coefficient among loci (see Simons et al., 2018), regardless of the number of traits under selection (otherwise, selection coefficients would increase with the number of traits under selection; Appendix A.1.3). Under this model of pleiotropy, we find less of a reduction in the prediction accuracy of ascertained variation compared to our baseline scenario. This is because the effect of the mutation on the trait of interest becomes more weakly correlated with its total selection coefficient. Specifically, common alleles can have larger effects on the trait of interest but by chance experience weaker selection due to small effects on the other selected traits. Polymorphisms in the common ancestor that are large effect yet weakly selected will go to fixation or loss more slowly in each descendant population, and contribute a higher variance to the trait of interest in each of them because it is more likely to segregate at intermediate frequencies. Thus pleiotropy, for a constant average strength of selection, reduces the rate at which prediction accuracy declines.

\section{Difference in polygenic means among populations}

In the previous section we described how stabilizing selection and ascertainment in the GWAS sample can reduce the prediction accuracy of individual genetic values, but what are the consequences for the mean polygenic score of populations? The mean polygenic score of the population is the sum of population allele frequencies weighted by effect sizes. If a trait is neutrally evolving, the loci contributing to its variation are just like other neutrally evolving loci, and so differences among populations in their mean polygenic score just reflect a weighted sum of neutral allele frequency differences. Naively as trait increasing alleles underlying a neutral trait are equally likely to drift up or down, one might think that over many loci we expect only a small mean difference between populations. However, the polygenic score is a sum rather than a mean, and so each locus we add into the score is like an additional step in the random walk that two populations take away from each other (Chakraborty and Nei, 1982). We expect the variance among populations, i.e. the average squared difference between population means and the global mean, to be $2 V_{A} F_{S T}$ (Wright, 1951; Lande, 1992). We first explore the differentiation of mean polygenic scores under a constant optimum and constant environment, and then relax these assumptions.

\subsection{Stabilizing selection to a constant optimum}

Under a constant selective environment, stabilizing selection keeps population mean phenotypes close to their optimum in the face of genetic drift and mutation, such that the difference in mean phenotypes among populations with the same optimum should be minor relative to neutral expectations (even accounting for the lower genetic variance within populations under stabilizing selection; Figure 3A). This reduction in 
the divergence of the mean phenotype between populations reflects the fact that if trait-increasing alleles accidentally drift up in frequency, thus pushing the population mean away from its selective optimum, trait-decreasing alleles are subject to directional selection in their favour (and vice versa).

To explore population differences in mean polygenic scores, we can use $Q_{X}$ (Berg and Coop, 2014), the polygenic score equivalent of $Q_{S T} / F_{S T}$ (Prout and Barker, 1993; Spitze, 1993). $Q_{S T}$ measures half of the proportion of total variance in additive genetic values attributable to among-population differences. Under neutrality, for strictly additive traits we expect $Q_{S T}$ to equal $F_{S T}$ estimated from neutral polymorphisms (Lande, 1992; Whitlock, 1999), and their ratio should be $\chi^{2}$-distributed with degrees of freedom equal to one fewer than the number of populations (an extension of the Lewontin-Krakauer test; Lewontin and Krakauer, 1973). The polygenic score analog is $Q_{X}$ (Berg and Coop, 2014), where additive genetic values are substituted by polygenic scores. For our pair of populations, our $Q_{X}$ statistic is

$$
Q_{X}=\frac{\left(Z_{A}-Z_{B}\right)^{2}}{4 V_{a} F_{S T}}
$$

where $Z$ is the mean polygenic score in the population denoted in the subscript. When population mean phenotypes are over-dispersed relative to neutral expectations, $Q_{X}$ will be larger than 1 , potentially resulting in a statistically significant p-value under the null distribution. When population means are under-dispersed relative to neutral expectations, as we would expect for traits under stabilizing selection with the same optimum across populations, $Q_{X}$ will be much smaller than 1 , and the p-values under the null will be large.

Stabilizing selection to the same optimum tightly constrains the difference in mean additive genetic values between populations, i.e. the difference in mean polygenic scores using all of the variation, to be much lower than the difference for neutral traits even after standardizing for the lower levels of variation (Figure 3A). This leads to a distribution of $Q_{X}$ that is skewed toward lower values than the the neutral $\chi^{2}$-distribution (Figure S8). However, when we ascertain the top 1\% of variance-contributing polymorphisms in population A, the level of standardized polygenic score differentiation under stabilizing selection becomes more similar to neutral levels (Figure 3B) with a comparable level of false positive signals of adaptive differentiation as in the neutral case (Figure S8). While this result is more specific to our choice of mutant effect size distribution and strengths of stabilizing selection, we can generally conclude that for cases of stabilizing selection with limited ascertainment, estimates of standardized mean polygenic score differences will approach and perhaps exceed what we observe under neutrality. Note that as expected by design of $Q_{X}$, under the neutral case, the distribution of standardized mean polygenic score differences stays about the same between ascertainment levels, whether ascertaining all polymorphisms from both populations or just the top $1 \%$ in population A. Thus stabilizing selection, combined with incomplete and asymmetric ascertainment, causes the inflation of estimated mean standardized population differences above that seen for the underlying genetic values.

Since under stabilizing selection to the same optimum we expect only small differences between populations in their mean genetic value, the disparity between the (true) differences in mean genetic values and (estimated) differences in mean polygenic scores represents the mean difference contributed by non-ascertained polymorphisms. Intuitively this occurs because if population B has a larger value of an ascertained polygenic score than population $\mathrm{A}$, then the ascertained trait-increasing alleles have by chance drifted up in population B (compared to A) and this imbalance will have induced directional selection for the rest of the trait-increasing alleles to decrease their frequency to keep the population close to the optimum (under high polygenicity). In line with our expectations, we find that the mean polygenic score differences calculated from ascertained sites and mean polygenic differences calculated from non-ascertained sites are close to opposite one another (Figure 3B). The countervailing effect of the non-ascertained loci is nosier when stabilizing selection is weaker, because with weaker selection population means can drift further from their optimum. These results confirm that mean polygenic scores calculated from all polymorphisms (i.e. additive genetic values) should closely match between populations experiencing stronger and similar selection pressures and highlight how incomplete ascertainment can by chance generate misleading differences between them.

\subsection{Adapting to a changing optimum or environment}

The combination of changes in the stabilizing selection regime and incomplete ascertainment of causal polymorphisms can also generate misleading differences in mean polygenic scores and signals of differential selection among populations. When the optimal phenotype shifts from its ancestral value equally in both 

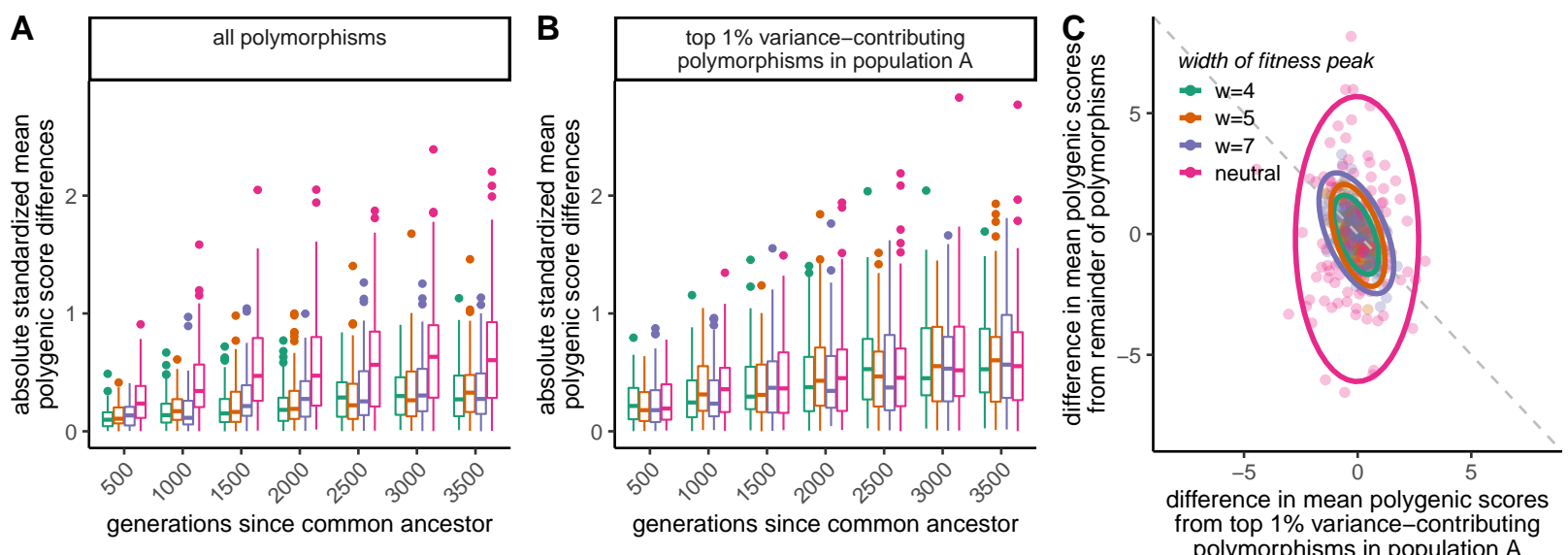

Figure 3: A) Absolute standardized mean polygenic score differences between populations A and B, $\frac{\left|Z_{A}-Z_{B}\right|}{\sqrt{V_{a}}}$, either when all polymorphisms in both populations were ascertained, or when the top $1 \%$ of variancecontributing polymorphisms in population A were ascertained. This measure has the same interpretation as $Q_{X}$; it equals $\sqrt{4 Q_{X} F_{S T}}$. B) Partitioning of mean polygenic score differences between the ascertained and non-ascertained set of polymorphisms. Ascertained polymorphisms are from top $1 \%$ of variance-contributing sites in population A. Points represent results for a single simulation. Ellipses denote the $95 \%$ confidence interval. The dashed grey line is the line of exactly opposing effects.

descendant populations, this directional selection generates equal responses in the true mean genetic values in each population that track each other extremely closely. However, because our polygenic score based on ascertained SNPs explains less of the variance in population B than population A, we capture less of its response to selection and thus artificially generate a difference between populations in their mean polygenic scores (Figure 4A). This shift in the mean polygenic score between populations, as well as a decrease in the total variance explained, can push the distribution of $Q_{X}$ towards larger values than the neutral case (Figure 4, Figure S12). The chances of getting a p-value below a significance threshold of 0.05 are highest when stabilizing selection is strongest $(w=4$; up to $20 \%$ chance depending on time since the optimum shift) but tend to be greater than neutrality for the strengths of selection we investigated (Figure S9). These signals of polygenic selection are consistent with the idea that $Q_{X}$ informs us about directional selection on polygenic scores, as selection has driven an increase in the polygenic score of A relative to B. However, they also highlight the very incomplete picture that we obtain about selection and genetic differences in the phenotype.

Under GxE, we also find higher chances of false signals of adaptation, even when the optimum never changes. The chances of these false signals decay with time since the common ancestor of the population pair (Figure S10). Under pleiotropy, we see similar, though slightly lower, chances for false signals of adaptation as under the baseline stabilizing selection scenario (Figure S11).

\section{Discussion}

Our work provides a foundation on which to understand how stabilizing selection on complex traits impacts the population differentiation of polygenic scores. We described our expectations for scenarios in which the optimum is the same among populations, which we treat as a null model for their evolution. Our work adds to recent investigations of how negative selection affects the prediction accuracy and portability of polygenic scores (Wang et al., 2020; Durvasula and Lohmueller, 2021). Here we focused explicitly on a model of quantitative trait evolution, which allowed us to better inform the relationship between an allele's phenotypic and fitness effects. By using this approach to model allele frequency dynamics, we gained insight into issues with portability and the interpretation of genetic differentiation of GWAS loci and polygenic scores among populations. 

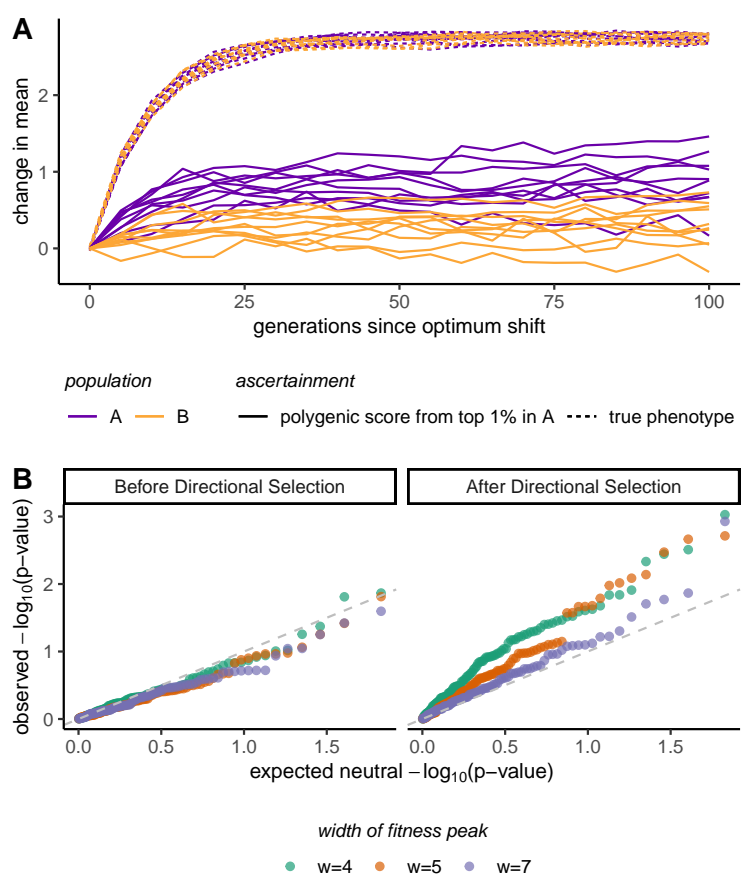

Figure 4: A) Change in mean phenotype or polygenic score over time since the optimum shifted 2 standard deviations of the phenotype distribution. True phenotypes are different from additive genetic values in that they account for the contribution of substitutions. B) Quantile-quantile plot of observed p-values against expected p-values under neutrality (uniform distribution). The dashed line shows equality; points that lie above this line indicate that the observed distribution has a higher density of low p-values than the neutral distribution, and points that lie below it indicate the opposite. 
While stabilizing selection with the same optimum among populations reduces phenotypic differentiation relative to neutrality, somewhat counter-intuitively it increases genetic differentiation at trait-influencing loci compared to neutral polymorphisms. The consequences of this differentiation at large effect QTLs and few loci have been explored by various authors (Latta, 1998; Le Corre and Kremer, 2003; Kremer and Le Corre, 2012), and here we investigated its implications in the age of GWAS. By increasing genetic differentiation at the loci underlying trait variation, stabilizing selection reduces the additive genic variance in one population that can be explained by polymorphisms in another. Thus polygenic scores constructed from polymorphisms ascertained in a GWAS will have reduced prediction accuracy in unrepresented populations compared to represented ones. The prediction accuracy for unrepresented populations decreases with increasing strengths of stabilizing selection and time since the common ancestor with represented populations. Occasional fluctuations in the fitness optima would negligibly influence our portability results, because such directional selection would cause very minor shifts in frequency over the short time scales we consider (Hayward and Sella, 2021).

The distribution of mutant effect sizes is critical to understanding the effects of stabilizing selection on the turnover of genetic variation among populations. With increasing weight towards larger mutant effects, to a certain extent stabilizing selection would cause a greater reduction in the shared additive genetic variance, and thus polygenic score portability. Conversely, if the trait was truly infinitesimal, stabilizing selection would not contribute to the loss of heterozygosity, such that the sharing of genetic variance would be well predicted by genetic drift, or neutral $F_{S T}$. While the loci mapped by GWAS are often of small effect, and the traits highly polygenic, we know that their effects are not infinitesimal, as loci must make a reasonable contribution to the variance to be discovered by a GWAS (see Simons et al., 2018, for a detailed population genetic model). While we do not consider migration between represented and unrepresented populations here, the general decline in portability with increasing genetic differentiation should hold, though the exact prediction will differ.

Pleiotropy and GxE complicate the predictions of stabilizing selection for portability. Differences in the environment among populations can lead to changes in the effect sizes of alleles (GxE), which weakens the portability as effect sizes will be only partially correlated between populations and stabilizing selection purges more of the ancestral variation shared between populations. Holding the average strength of selection on an allele constant, when alleles can independently affect multiple traits under stabilizing selection, the correlation between their effect size on the trait of interest and their selection coefficient weakens. Thus stabilizing selection purges less of shared variation and causes a weaker reduction in portability.

Like much of the recent work on portability, our work emphasizes the reduction in prediction accuracy for populations not represented in GWAS (Márquez-Luna, 2017; Martin et al., 2017a; Duncan et al., 2019; Lam et al., 2019; Weissbrod et al., 2021). A natural conclusion is that polygenic predictions that work well across populations will require GWAS across a range of diverse ancestries, in line with other calls to reduce Eurocentrism in GWAS (Li and Keating, 2014; Popejoy and Fullerton, 2016; Martin et al., 2019). In addition to changes in allele frequency and linkage disequilibrium, the relationship of polygenic scores to phenotypes will vary across populations due to variation in genetic effects, assortative mating, and differences in GxG and GxE. Indeed the prediction accuracy of polygenic scores for some traits was recently shown to be quite variable across different groups within an ancestry, suggesting that GxE and environmental variation are quite prevalent for some traits (Mostafavi et al., 2020). Thus, we caution that a better understanding of the portability of polygenic scores across populations will also need a stronger understanding of the causes of variation in prediction accuracy within populations.

With the rise of ancient DNA sequencing, GWAS from contemporary populations have also been used to construct polygenic scores for ancient individuals (reviewed in Irving-Pease et al., 2021). These scores have been used to provide a window into the phenotypic diversity of past populations (Mathieson et al., 2015; Berens et al., 2017; Martiniano et al., 2017) and to disentangle genetic and environmental contributors to temporal phenotypic variation (e.g. at the Neolithic transition; Cox et al., 2019, 2021; Marciniak et al., 2021). Such studies are most convincing when there are relevant phenotypic measurements on at least some ancient individuals and polygenic prediction accuracies can be judged. However, investigators will often not have this luxury, leaving unclear the insight these approaches can provide. Some studies using ancient DNA have identified reduced rates of disease alleles in the past compared to present-day populations. While we have focused here on quantitative traits, rather than disease traits, we caution that purifying selection against risk alleles will lead modern day populations to systematically underrepresent the diversity of disease 
alleles in the past (Berens et al., 2017; Aris-Brosou, 2019; Esteller-Cucala et al., 2020; Simonti and Lachance, 2021).

When using polygenic scores constructed from the ascertained set of polymorphisms, we increase the possibility of generating misleading signals of differentiation between populations. Stabilizing selection to a constant optimum alone does not generate more false signals of directional selection than what we expect under the neutral evolution of complex traits. However, the standardized difference between populations will be systematically over-estimated with ascertained polygenic scores, have standardized for the lower proportion of the genetic variance is explained. We see this result because with stabilizing selection, the unascertained portion of the variance tends to act exactly counter to the trend seen in the ascertained portion. Misleading signals of adaptive polygenic differentiation can also be generated when the stabilizing selection regime shifts in the same way in each population (such that there is still minor phenotypic differentiation between them) as the ascertained polymorphisms only capture a shift in the ascertainment population. This issue arises because stabilizing selection lowers the proportion of the additive genic variance explained in the non-GWAS population and so we capture a lower proportion of that population's response to directional selection. Thus this issue of missing the parallel adaptive response across ancestries can be expected in many situations with imperfect portability. Such signals of polygenic adaptation can be useful as $Q_{X}$ is correctly detecting that directional selection has acted on the genetic variation along the branch leading to the GWAS population, but the signal is very open to the misinterpretation that the non-GWAS population has not also responded to the same selection pressures.

Many traits have likely experienced a mixture of stabilizing selection and bursts of directional selection across human history. Even if the populations share the same phenotypic optimum, if an environmental change systematically shifts one population away from this optimum, there would be directional polygenic adaptation to move that population back towards the optimum, resulting in a difference in polygenic scores but no difference in the mean phenotypes between populations (Harpak and Przeworski, 2021). Therefore, under current ascertainment schemes and pervasive stabilizing selection, the difference in mean polygenic scores among populations provides very unreliable information about the potential role of selection in generating phenotypic differences among populations.

A polygenic score is a prediction of how an individual's phenotype is expected to deviate away from the sample mean given their genotype at some (large) number of polymorphisms. Sometimes they are quoted as absolute values, but that is always based on an empirical phenotypic mean. The mean polygenic score in a population cannot inform us of the mean phenotype in a population, even if it was constructed from all polymorphisms and averaged across all individuals. This is because these scores are based on polymorphic genotypes alone whereas the absolute phenotype of an individual represents the end product of the entire genome and environment played out through a vast number of developmental processes. Yet it is easy to fall into the trap of believing that a difference in polygenic scores between groups is a strong statement about the difference in the mean phenotype of those groups who also differ in a myriad of environmental and cultural factors (a related set of issues are present in epidemology; Rose, 2001). Thus while the field of human genetics is increasing its power to predict phenotypic variance among individuals within groups, it remains a poor guide to the causes of phenotypic variance among groups with greater environmental and genetic differentiation (Lewontin, 1974; Feldman and Lewontin, 1975).

\section{Acknowledgements}

We thank Doc Edge, Molly Przeworski, and Guy Sella for their valuable comments on earlier drafts of our manuscript. We also thank the members of the Coop lab for helpful discussions. Funding was provided by the National Institute of General Medical Sciences of the National Institutes of Health (NIH R01 GM108779 and R35 GM136290, awarded to GC).

\section{References}

Adhikari, K., J. Mendoza-Revilla, A. Sohail, M. Fuentes-Guajardo, J. Lampert, et al., 2019 A GWAS in Latin Americans highlights the convergent evolution of lighter skin pigmentation in Eurasia. Nature Communications 10. 
Aris-Brosou, S., 2019 Direct Evidence of an Increasing Mutational Load in Humans. Molecular Biology and Evolution 36: 2823-2829.

Balding, D. J. and R. A. Nichols, 1994 Dna profile match probability calculation: how to allow for population stratification, relatedness, database selection and single bands. Forensic Science International 64: 125-140.

Barbujani, G., A. Magagni, E. Minch, and L. L. Cavalli-Sforza, 1997 An apportionment of human dna diversity. Proceedings of the National Academy of Sciences 94: 4516-4519.

Barton, N. H. and P. D. Keightley, 2002 Understanding quantitative genetic variation. Nature Reviews Genetics 3: 11-21.

Bentley, A. R., Y. J. Sung, M. R. Brown, T. W. Winkler, A. T. Kraja, et al., 2019 Multi-ancestry genomewide gene-smoking interaction study of 387,272 individuals identifies new loci associated with serum lipids. Nature Genetics 51: 636-648.

Berens, A. J., T. L. Cooper, and J. Lachance, 2017 The genomic health of ancient hominins. Human Biology 89: $7-19$.

Berg, J. J. and G. Coop, 2014 A Population Genetic Signal of Polygenic Adaptation. PLoS Genetics 10: e1004412.

Berg, J. J., A. Harpak, N. Sinnott-Armstrong, A. M. Joergensen, H. Mostafavi, et al., 2019 Reduced signal for polygenic adaptation of height in UK Biobank. eLife 8: e39725.

Bergström, A., S. A. McCarthy, R. Hui, M. A. Almarri, Q. Ayub, et al., 2020 Insights into human genetic variation and population history from 929 diverse genomes. Science 367: eaay5012.

Boyle, E. A., Y. I. Li, and J. K. Pritchard, 2017 An Expanded View of Complex Traits: From Polygenic to Omnigenic. Cell 169: 1177-1186.

Brown, B. C., A. Genetic, E. Network, T. Diabetes, C. J. Ye, et al., 2016 Transethnic Genetic-Correlation Estimates from Summary Statistics. The American Journal of Human Genetics 99: 76-88.

Bulmer, M., 1971 The Effect of Selection on Genetic Variability. The American Naturalist 105: 201-211.

Bumpus, H., 1899 The Elimination of the Unfit as Illustrated by the Introduced Sparrow, Passer Domesticus: (a Fourth Contribution to the Study of Variation). Biological lectures delivered at the Marine Biological Laboratory of Wood's Hole, Gin.

Cavazos, T. B. and J. S. Witte, 2021 Inclusion of variants discovered from diverse populations improves polygenic risk score transferability. Human Genetics and Genomics Advances 2: 100017.

Chakraborty, R., 1990 Quantitative Traits in Relation to Population Structure: Why and How Are They Used and What Do They Imply? Human Biology 62: 147-162.

Chakraborty, R. and M. Nei, 1982 Genetic differentiation of quantitative characters between populations or species I: Mutation and random genetic drift. Genetical Research 39: 303-314.

Conrad, D. F., M. Jakobsson, G. Coop, X. Wen, J. D. Wall, et al., 2006 A worldwide survey of haplotype variation and linkage disequilibrium in the human genome. Nature Genetics 38: 1251-1260.

Conti, D. V., B. F. Darst, L. C. Moss, E. J. Saunders, X. Sheng, et al., 2021 Trans-ancestry genome-wide association meta-analysis of prostate cancer identifies new susceptibility loci and informs genetic risk prediction. Nature Genetics 53: 65-75.

Coop, G., 2019 Reading tea leaves? polygenic scores and differences in traits among groups. arXiv preprint arXiv:1909.00892 .

Coop, G., J. K. Pickrell, J. Novembre, S. Kudaravalli, J. Li, et al., 2009 The Role of Geography in Human Adaptation. PLoS Genetics 5: e1000500. 
Coram, M. A., H. Fang, S. I. Candille, T. L. Assimes, and H. Tang, 2017 Leveraging Multi-ethnic Evidence for Risk Assessment of Quantitative Traits in Minority Populations. The American Journal of Human Genetics 101: 218-226.

Cox, S. L., H. Moots, J. T. Stock, A. Shbat, B. D. Bitarello, et al., 2021 Predicting skeletal stature using ancient DNA. bioRxiv .

Cox, S. L., C. B. Ruff, R. M. Maier, and I. Mathieson, 2019 Genetic contributions to variation in human stature in prehistoric Europe. Proceedings of the National Academy of Sciences 116: 201910606.

Curtis, D., 2018 Polygenic risk score for schizophrenia is more strongly associated with ancestry than with schizophrenia. Psychiatric Genetics 28: 85-89.

de Villemereuil, P., A. Charmantier, D. Arlt, P. Bize, P. Brekke, et al., 2020 Fluctuating optimum and temporally variable selection on breeding date in birds and mammals. Proceedings of the National Academy of Sciences 117: 31969-31978.

Duncan, L., H. Shen, B. Gelaye, J. Meijsen, K. Ressler, et al., 2019 Analysis of polygenic risk score usage and performance in diverse human populations. Nature Communications 10.

Durvasula, A. and K. E. Lohmueller, 2021 Negative selection on complex traits limits phenotype prediction accuracy between populations. The American Journal of Human Genetics 108: 620-631.

Edge, M. D. and N. A. Rosenberg, 2015 A general model of the relationship between the apportionment of human genetic diversity and the apportionment of human phenotypic diversity. Human Biology 87: 313-337.

Esteller-Cucala, P., I. Maceda, A. D. Børglum, D. Demontis, S. V. Faraone, et al., 2020 Genomic analysis of the natural history of attention-deficit/hyperactivity disorder using Neanderthal and ancient Homo sapiens samples. Scientific Reports 10.

Fan, S., M. E. Hansen, Y. Lo, and S. A. Tishkoff, 2016 Going global by adapting local: A review of recent human adaptation. Science 354: 54-59.

Feldman, M. W. and R. C. Lewontin, 1975 The Heritability Hang-up. Science 190: 1163-1168.

Galinsky, K. J., Y. A. Reshef, H. K. Finucane, P. R. Loh, N. Zaitlen, et al., 2019 Estimating cross-population genetic correlations of causal effect sizes. Genetic Epidemiology 43: 180-188.

Gingerich, P. D., 1983 Rates of evolution: Effects of time and temporal scaling. Science 222: 159-161.

Goldstein, D. B. and K. E. Holsinger, 1992 Maintenance of polygenic variation in spatially structured populations: roles for local mating and genetic redundancy. Evolution 46: 412-429.

Grinde, K. E., T. A. Thornton, K. H. K. Chan, and A. P. Reiner, 2019 Generalizing polygenic risk scores from Europeans to Hispanics / Latinos. Genetic Epidemiology 43: 50-62.

Haller, B. C. and P. W. Messer, 2019 SLiM 3: Forward Genetic Simulations Beyond the Wright-Fisher Model. Molecular Biology and Evolution 36: 632-637.

Harpak, A. and M. Przeworski, 2021 The evolution of group differences in changing environments. PLoS Biology 19: e3001072.

Haworth, S., R. Mitchell, L. Corbin, K. H. Wade, T. Dudding, et al., 2019 Apparent latent structure within the UK Biobank sample has implications for epidemiological analysis. Nature Communications 10.

Hayward, L. K. and G. Sella, 2021 Polygenic adaptation after a sudden change in environment. bioRxiv .

Hernandez, R. D., J. L. Kelley, E. Elyashiv, S. C. Melton, A. Auton, et al., 2011 Classic Selective Sweeps Were Rare in Recent Human Evolution. Science 257: 920-924. 
Hill, W. G., M. E. Goddard, and P. M. Visscher, 2008 Data and theory point to mainly additive genetic variance for complex traits. PLOS Genetics 4: 1-10.

Hill, W. G. and M. Kirkpatrick, 2010 What animal breeding has taught us about evolution. Annual Review of Ecology, Evolution, and Systematics 41: 1-19.

Horikoshi, M., T. Sofer, A. Mahajan, H. Kitajima, N. Franceschini, et al., 2017 Trans-ethnic meta-regression of genome-wide association studies accounting for ancestry increases power for discovery and improves fine-mapping resolution. Human Molecular Genetics 26: 3639-3650.

Houle, D., G. H. Bolstad, K. Van Der Linde, and T. F. Hansen, 2017 Mutation predicts 40 million years of fly wing evolution. Nature 548: 447-450.

Irving-Pease, E. K., R. Muktupavela, M. Dannemann, and F. Racimo, 2021 Quantitative Human Paleogenetics: what can ancient DNA tell us about complex trait evolution? Frontiers in Genetics 12: 703541.

Isshiki, M., Y. Watanabe, and J. Ohashi, 2021 Geographic variation in the polygenic score of height in Japan. Human Genetics 140: 1097-1108.

Jorde, L. B., W. S. Watkins, M. J. Bamshad, M. Dixon, C. Ricker, et al., 2000 The distribution of human genetic diversity: a comparison of mitochondrial, autosomal, and y-chromosome data. The American Journal of Human Genetics 66: 979-988.

Keightley, P. D. and W. G. Hill, 1988 Quantitative genetic variability maintained by mutation-stabilizing selection balance in finite populations. Genetical Research 52: 33-43.

Kerminen, S., A. R. Martin, J. Koskela, S. E. Ruotsalainen, A. S. Havulinna, et al., 2019 Geographic Variation and Bias in the Polygenic Scores of Complex Diseases and Traits in Finland. American Journal of Human Genetics 104: 1169-1181.

Kim, M. S., K. P. Patel, A. K. Teng, A. J. Berens, and J. Lachance, 2018 How genetic disease risks can be misestimated across global populations. Genome Biology 19.

Kingsolver, J. G., H. E. Hoekstra, J. M. Hoekstra, D. Berrigan, S. N. Vignieri, et al., 2001 The strength of phenotypic selection in natural populations. The American Naturalist 157: 245-261.

Kremer, A. and V. Le Corre, 2012 Decoupling of differentiation between traits and their underlying genes in response to divergent selection. Heredity 108: 375-385.

Lam, M., C.-y. Chen, Z. Li, A. R. Martin, and J. Bryois, 2019 Comparative genetic architectures of schizophrenia in East Asian and European populations. Nature Genetics 51.

Lande, R., 1976 Natural selection and random genetic drift in phenotypic evolution. Evolution 30: 314-334.

Lande, R., 1991 Isolation by distance in a quantitative trait. Genetics 128: 443-452.

Lande, R., 1992 Neutral Theory of Quantitative Genetic Variance in an Island Model with Local Extinction and Colonization 46: 381-389.

Lande, R. and S. J. Arnold, 1983 The measurement of selection on correlated characters. Evolution 37: $1210-1226$.

Latta, R. G., 1998 Differentiation of allelic frequencies at quantitative trait loci affecting locally adaptive traits. American Naturalist 151: 283-292.

Le Corre, V. and A. Kremer, 2003 Genetic Variability at Neutral Markers, Quantitative Trait Loci and Trait. Genetics 1219: 1205-1219.

Lewontin, R. C., 1972 The Apportionment of Human Diversity. In Evolutionary Biology, edited by T. Dobzhansky, M. K. Hecht, and W. C. Steere, chapter 14, pp. 381-398, Appleton-Century-Crofts, New York. 
Lewontin, R. C., 1974 The analysis of variance and the analysis of causes. American Journal of Human Genetics 26: 400-411.

Lewontin, R. C. and J. Krakauer, 1973 Distribution of gene frequency as a test of the theory of the selective neutrality of polymorphisms. Genetics 74: 175-195.

Li, J. Z., D. M. Absher, H. Tang, A. M. Southwick, A. M. Casto, et al., 2008 Worldwide human relationships inferred from genome-wide patterns of variation. Science 319: 1100-1104.

Li, Y. R. and B. J. Keating, 2014 Trans-ethnic genome-wide association studies: advantages and challenges of mapping in diverse populations 6 .

Liu, J. Z., S. Van Sommeren, H. Huang, S. C. Ng, R. Alberts, et al., 2015 Association analyses identify 38 susceptibility loci for inflammatory bowel disease and highlight shared genetic risk across populations. Nature Genetics 47: 979-986.

Loh, P. R., G. Bhatia, A. Gusev, H. K. Finucane, B. K. Bulik-Sullivan, et al., 2015 Contrasting genetic architectures of schizophrenia and other complex diseases using fast variance-components analysis. Nature Genetics 47: 1385-1392.

Macarthur, J., E. Bowler, M. Cerezo, L. Gil, P. Hall, et al., 2017 The new NHGRI-EBI Catalog of published genome-wide association studies (GWAS Catalog). Nucelic Acids Research 45: 896-901.

Marciniak, S., C. M. Bergey, A. M. Silva, A. Hałuszko, M. Furmanek, et al., 2021 An integrative skeletal and paleogenomic analysis of prehistoric stature variation suggests relatively reduced health for early European farmers. bioRxiv .

Márquez-Luna, C., 2017 Multiethnic polygenic risk scores improve risk prediction in diverse populations. Genetic Epidemiology 41: 811-823.

Martin, A. R., C. R. Gignoux, R. K. Walters, G. L. Wojcik, B. M. Neale, et al., 2017a Human Demographic History Impacts Genetic Risk Prediction across Diverse Populations. The American Journal of Human Genetics 100: 635-649.

Martin, A. R., M. Kanai, Y. Kamatani, Y. Okada, B. M. Neale, et al., 2019 Clinical use of current polygenic risk scores may exacerbate health disparities. Nature Genetics 51: 584-591.

Martin, A. R., M. Lin, J. M. Granka, J. W. Myrick, X. Liu, et al., 2017b An Unexpectedly Complex Architecture for Skin Pigmentation in Africans. Cell 171: 1340-1353.

Martiniano, R., L. M. Cassidy, R. Ó’Maoldúin, R. McLaughlin, N. M. Silva, et al., 2017 The population genomics of archaeological transition in west Iberia: Investigation of ancient substructure using imputation and haplotype-based methods. PLoS Genetics 13: 1-24.

Maruyama, T. and M. Kimura, 1975 Moments for Sum of an Arbitrary Function of Gene Frequency along a Stochastic Path of Gene Frequency Change. Proceedings of the National Academy of Sciences 72: 16021604 .

Mathieson, I., 2021 The omnigenic model and polygenic prediction of complex traits. The American Journal of Human Genetics 108: 1-6.

Mathieson, I., I. Lazaridis, N. Rohland, S. Mallick, N. Patterson, et al., 2015 Genome-wide patterns of selection in 230 ancient Eurasians. Nature 528: 499-503.

Mostafavi, H., A. Harpak, I. Agarwal, D. Conley, J. K. Pritchard, et al., 2020 Variable prediction accuracy of polygenic scores within an ancestry group. eLife 9: e48376.

Narain, P. and R. Chakraborty, 1987 Genetic differentiation of quantitative characters between populations or species II: Optimal selection in infinite populations. Heredity 59: 199-212. 
Novembre, J. and N. H. Barton, 2018 Tread lightly interpreting polygenic tests of selection. Genetics 208: $1351-1355$.

Popejoy, A. B. and S. M. Fullerton, 2016 Genomics is failing on diversity. Nature 538: 161-164.

Prout, T. and J. S. F. Barker, 1993 F Statistics in Drosophila buzzatii: Selection, Population Size and Inbreeding. Genetics 134: 369-375.

Refoyo-Martínez, A., S. Liu, A. M. Jørgensen, X. Jin, A. Albrechtsen, et al., 2020 How robust are crosspopulation signatures of polygenic adaptation in humans? bioRxiv pp. 1-66.

Relethford, J. H. and F. C. Lees, 1982 The use of quantitative traits in the study of human population structure. American Journal of Physical Anthropology 25: 113-132.

Robertson, A., 1956 The effect of selection against extreme deviants based on deviation or on homozygosis. Journal of Genetics 54: 236-248.

Rogers, A. R. and H. C. Harpending, 1983 Population structure and quantitative characters. Genetics 105: 985-1002.

Rose, G., 2001 Sick individuals and sick populations. International Journal of Epidemiology 30: 396-427-432.

Rosenberg, N. A., M. D. Edge, J. K. Pritchard, and M. W. Feldman, 2019 Interpreting polygenic scores, polygenic adaptation, and human phenotypic differences. Evolution, medicine, and public health 2019: $26-34$.

Rosenberg, N. A., J. K. Pritchard, J. L. Weber, H. M. Cann, K. K. Kidd, et al., 2002 Genetic structure of human populations. Science 298: 2381-2385.

Sakaue, S., M. Kanai, Y. Tanigawa, J. Karjalainen, M. Kurki, et al., 2020 A global atlas of genetic associations of 220 deep phenotypes. medRxiv pp. 1-52.

Sanjak, J. S., J. Sidorenko, M. R. Robinson, K. R. Thornton, and P. M. Visscher, 2018 Evidence of directional and stabilizing selection in contemporary humans. Proceedings of the National Academy of Sciences 115: $151-156$.

Sawyer, S. A. and D. L. Hartl, 1992 Population Genetics of Polymorphism and Divergence. Genetics 132: 1161-1176.

Sella, G. and N. H. Barton, 2019 Thinking about the evolution of complex traits in the era of genome-wide association studies. Annual Review of Genomics and Human Genetics 20: 461-493.

Shi, H., G. Kichaev, and B. Pasaniuc, 2016 Contrasting the Genetic Architecture of 30 Complex Traits from Summary Association Data. American Journal of Human Genetics 99: 139-153.

Simons, Y. B., K. Bullaughey, R. R. Hudson, and G. Sella, 2018 A population genetic interpretation of GWAS findings for human quantitative traits. PLoS Biology 16.

Simonti, C. N. and J. Lachance, 2021 Ancient DNA reveals that few GWAS loci have been strongly selected during recent human history. bioRxiv .

Sohail, M., R. M. Maier, A. Ganna, A. Bloemendal, A. R. Martin, et al., 2019 Polygenic adaptation on height is overestimated due to uncorrected stratification in genome-wide association studies. eLife 8: e39702.

Spitze, K., 1993 Population structure in Daphnia obtusa: Quantitative genetic and allozymic variation. Genetics 135: 367-374.

Trochet, H. and J. Hussin, 2020 Fine-scale population structure confounds genetic risk scores in the ascertainment population. bioRxiv .

Turchin, M. C., C. W. Chiang, C. D. Palmer, S. Sankararaman, D. Reich, et al., 2012 Evidence of widespread selection on standing variation in europe at height-associated snps. Nature Genetics 44: 1015-1019. 
Turelli, M. and N. Barton, 1990 Dynamics of polygenic characters under selection. Theoretical Population Biology 38: 1-57.

Veturi, Y., N. Yi, W. Huang, and A. I. Vazquez, 2019 Modeling Heterogeneity in the Genetic Architecture of Ethnically Diverse Groups Using Random Effect Interaction Models. Genetics 211: 1395-1407.

Vilhjálmsson, B. J., J. Yang, H. K. Finucane, A. Gusev, S. Ripke, et al., 2015 Modeling Linkage Disequilibrium Increases Accuracy of Polygenic Risk Scores. American Journal of Human Genetics 97: 576-592.

Wang, Y., J. Guo, G. Ni, J. Yang, P. M. Visscher, et al., 2020 Theoretical and empirical quantification of the accuracy of polygenic scores in ancestry divergent populations. Nature Communications 11: 1-9.

Weber, K. E., 1990 Selection on wing allometry in Drosophila melanogaster. Genetics 126: 975-989.

Weissbrod, O., M. Kanai, H. Shi, S. Gazal, W. Peyrot, et al., 2021 Leveraging fine-mapping and nonEuropean training data to improve trans-ethnic polygenic risk scores. medRxiv .

Whitlock, M. C., 1999 Neutral additive genetic variance in a metapopulation. Genetics Research 74: 215-221.

Wojcik, G. L., M. Graff, K. K. Nishimura, R. Tao, J. Haessler, et al., 2019 Genetic analyses of diverse populations improves discovery for complex traits. Nature 570: 514-518.

Wright, S., 1935 Evolution in populations in approximate equilibrium. Journal of Genetics 30: 257-266.

Wright, S., 1951 The Genetical Structure of Populations. Annals of Eugenics 15: 323-354. 


\section{A Appendix}

\section{A.1 Simulation Details}

In SLiM version 3.6 (Haller and Messer, 2019), we simulated stabilizing selection on additive traits and divergence between a pair of populations with the same optimal phenotype (set at zero). Each simulation consisted of a constant population size of 10,000 diploid individuals and genomes comprising $30001 \mathrm{~kb}$ quantitative trait loci and 1000 1kb neutral loci, with free recombination between loci and a per base pair recombination rate of $10^{-8}$ within loci. Mutations arose at rate $10^{-8}$ per base pair and for quantitative trait loci their effect size was randomly assigned from a normal distribution. We used a Gaussian fitness function setting the fitness of an individual to

$$
W(\vec{r})=\exp \left(-\frac{r^{2}}{2 w^{2}}\right),
$$

where $r=\|\vec{r}\|$ is the phenotypic distance from the optimum of an individual with a vector of phenotypes $\vec{r}$ and $w$ quantifies the width of the fitness peak. The form of Gaussian selection is consistent with any form of quadratic selection if the population mean is near the optimum (see section A.1.2). We simulated quantitative traits under neutrality $(w=\infty)$ and under varying widths of the fitness peak, $w$, in which larger values of $w$ indicate weaker stabilizing selection. We chose strengths of stabilizing selection and an effect size distribution that correspond to observations in human populations (see section A.1.1 and A.1.2 for details). We calculated an individual's fitness each generation based on equation A.1, where their phenotype was the sum of the effects of alleles they carried at both variable and fixed sites.

We burned in each simulation for $60,000(6 N)$ generations before splitting the population into two for 3500 generations. For every 500 generations of population divergence, we recorded mutation effect sizes and frequencies in each population. To reduce computation times, groups of 10 simulation replicates shared the first 40,000 generations of their burn-in. The remaining 20,000 generations provided more than enough time for a complete turnover in the genetic basis of trait variation, such that simulations within a group were effectively independent. We simulated 100 replicates of each parameter combination.

\section{A.1.1 Choice of parameter values for strength of selection on the trait}

We simulated under four different strengths of stabilizing selection $(w)$, chosen to range from no selection (neutral trait) to the highest strength of stabilizing selection estimated from the UK BioBank (Sanjak et al., 2018). These estimates were determined from regressions of relative fitness (based on estimates of lifetime reproductive success) on squared standard normal phenotypes, $r^{2}$, where half of the coefficient of the quadratic term is the quadratic selection gradient, $\gamma$, in which negative values imply stabilizing selection, and the intercept is the mean fitness in the population, $\bar{w}$. The authors report estimates of $\gamma$ from their regressions, and so to get strengths of stabilizing selection $(w)$ under our slightly different fitness model, we make the following approximations to fitness. At equilibrium, the distribution of phenotypes are closely centered around the optimal phenotype relative to the width of the fitness gradient, and therefore we assume that mean fitness $\bar{w} \approx 1$. Additionally assuming that the mean phenotype in the population is the optimal phenotype, we can then approximate that the quadratic fitness function that is equivalent to ours when $\gamma=-\frac{1}{w^{2}}$, because $\frac{\gamma}{2} r^{2}+1 \approx \exp \left(\frac{\gamma r^{2}}{2}\right)$. Based on incremental $\gamma$ values of $0,-0.02,-0.04$, and -0.06 (the maximum estimated), we use the following values of the strength of stabilizing selection on the trait: $w=\infty$ (neutral trait), $w=7, w=5$, and $w=4$.

\section{A.1.2 Choice of effect size distribution}

Effect sizes of new mutations were drawn from a normal distribution with mean zero. Provided that the strengths of selection we chose correspond to selection on standard normal phenotypes, we used a standard deviation that would result in a genetic variance close to 1 at equilibrium. See equation A.16 for the solution for the equilibrium genic variance. Since the strength of selection determines the equilibrium genic variance, thus leading to different solutions for the variance of effect sizes of incoming mutations, we used the intermediate strength of selection to determine the variance of this effect size distribution $(w=5)$. We used standard deviations $\left(\sigma_{a}\right)$ of 0.05 and 0.1 . All results in the main text correspond to the standard deviation of 0.1 . 


\section{A.1.3 Extensions}

Directional selection We extended our baseline stabilizing scenario for directional selection by imposing a positive optimum shift of either one or two standard deviations of the phenotypic distribution. The optimum shifted 2500 generations after the pair of populations diverged. We started directional selection simulations from the states of the baseline scenario that were recorded at the time of the optimum shift. To calculate the extent of the optimum shift in each simulation, we averaged the phenotypic standard deviation of each population in that simulation.

Gene-by-environment interactions (GxE) We simulated GxE using the same framework as the baseline scenario, except we drew the effect sizes of mutations from a multivariate normal distribution with three dimensions representing the ancestral burn-in population and its two descendant populations. The covariance matrix was determined according to the standard deviation of the effect size distribution and correlation of effect sizes provided (either 0.9 or 0.95 ). Phenotypes and thus fitness were calculated using the effect size assigned for the particular population in which they were evaluated. The effect size correlations we chose are higher than most estimates, though GxG and differences in LD likely play a role as well (Liu et al., 2015; Galinsky et al., 2019; Lam et al., 2019; Veturi et al., 2019).

Pleiotropy We simulated pleiotropy using the same framework as the baseline scenario. A mutation's effect on each trait was independently drawn from the same normal distribution. An individual's fitness was calculated using their Euclidean distance from the optimum, based on their distance in each phenotypic dimension. For this extension we simulated $n=5$ and $n=10$ traits (the baseline scenario is $n=1$ ). The selection coefficient that an allele experiences is approximately

$$
s \approx \frac{\sum_{i=1}^{n} a_{i}^{2}}{w^{2}}
$$

where $a_{i}$ denotes the allele's effect on trait $i$. Thus when we sample the effect of an allele on each trait independently and use the same distribution, with more pleiotropy there would always be stronger selection, where

$$
\mathrm{E}(s)=\frac{n \operatorname{Var}\left(a_{i}\right)}{w^{2}} .
$$

As it is more realistic that each trait is more weakly selected under pleiotropy, we standardized the fitness function so that the average selection coefficient would be the same across pleiotropy levels $(n)$. To achieve the same expectation across simulations, we multiplied $w^{2}$ by $n$ in the fitness function. While the average selection coefficient is the same for a particular $w$ across $n$, its distribution differs according to the number of traits influenced,

$$
s \sim \frac{\operatorname{Var}\left(\mathrm{a}_{\mathrm{i}}\right)}{n w^{2}} \chi_{n}^{2} .
$$

\section{A.2 Relative prediction accuracy of polygenic scores}

The prediction accuracy of a polygenic score is the the proportion of the phenotypic variance explained by that polygenic score (the squared correlation $R^{2}$ ). We write out the correlation between an individual's polygenic score constructed from unlinked polymorphisms discovered by GWAS, $P_{i}$, and the true additive genetic value, $G_{i}$, in a particular target population. We assume that a GWAS discovers associations only at causal loci, and that at these loci with significant associations, it estimates their true effects. There are $L_{s}$ loci with significant associations in the study and a remaining $L_{r}$ that contribute to trait variation in a 
particular target population. Therefore an individual's predicted and true genetic values are

$$
\begin{aligned}
P_{i} & =\sum_{l=1}^{L_{s}} a_{l} g_{i l} \\
G_{i} & =\sum_{l=1}^{L_{s}+L_{r}} a_{l} g_{i l} \\
& =P_{i}+G_{i}^{(r)}, \text { where } G_{i}^{(r)}=\sum_{l=L_{s}+1}^{L_{s}+L_{r}} a_{l} g_{i l},
\end{aligned}
$$

in which their true genetic value is a sum of their predicted genetic value and genetic value contributed by the remainder of sites contributing to trait variation. Because the predicted genetic value is a portion of the true genetic value, the covariance between them is the variance in predicted genetic values,

$$
\begin{aligned}
\operatorname{Cov}(P, G) & =\operatorname{Cov}\left(P, P+G_{i}^{(r)}\right) \\
& =\operatorname{Cov}(P, P) \\
& =\operatorname{Var}(P)
\end{aligned}
$$

Thus the correlation between the predicted and true genetic values is

$$
\operatorname{Corr}(P, G)=\frac{\operatorname{Var}(P)}{\sqrt{\operatorname{Var}(P) \times \operatorname{Var}(G)}}=\sqrt{\frac{\operatorname{Var}(P)}{\operatorname{Var}(G)}},
$$

meaning that the correlation between the predicted and true additive genetic values is the square root of the proportion of the trait variance explained by SNPs ascertained by the GWAS, or that the variance in true genetic values explained by polygenic scores $\left(r^{2}\right)$ is the proportion of the variance explained by the polymorphisms used to construct the polygenic scores. This value also represents the reduction in the prediction accuracy of polygenic scores compared to additive genetic values, when predicting true additive phenotypes (with contributions from both genetics and environment). We denote the additive environmental contribution to the phenotype in an individual as $E_{i}$. The prediction accuracy of an individual's full additive phenotype $\left(G_{i}+E_{i}\right)$ when using polygenic scores is

$$
\begin{aligned}
r_{P}^{2} & =\frac{\operatorname{Cov}^{2}(P, G+E)}{\operatorname{Var}(P) \operatorname{Var}(G+E)} \\
& =\frac{\operatorname{Var}^{2}(P)}{\operatorname{Var}(P) \operatorname{Var}(G+E)} \\
& =\frac{\operatorname{Var}(P)}{\operatorname{Var}(G+E)}
\end{aligned}
$$

and when using additive genetic values is

$$
\begin{aligned}
r_{G}^{2} & =\frac{\operatorname{Cov}^{2}(G, G+E)}{\operatorname{Var}(G) \operatorname{Var}(G+E)} \\
& =\frac{\operatorname{Var}(G)}{\operatorname{Var}(G+E)},
\end{aligned}
$$

such that the reduction of prediction accuracy when using polygenic scores instead of additive genetic values is

$$
\frac{r_{P}^{2}}{r_{G}^{2}}=\frac{\operatorname{Var}(P)}{\operatorname{Var}(G)}
$$

Thus $r^{2}$ represents the prediction accuracy of polygenic scores for additive genetic values, as well as the reduction in prediction accuracy for full additive phenotypes due to incomplete ascertainment. 


\section{A.2.1 Modification for gene-by-environment interactions}

While we assume the effects of a causal allele are perfectly estimated in the GWAS sample, these effects may differ in populations that experience different environments (GxE). Thus for populations not represented in the GWAS, the correlation between polygenic scores and true additive genetic values will be lower than without GxE. Imagine that we use the set of effect sizes from GWAS population $A\left(a_{l, A}\right)$ to construct polygenic scores for population $B$ where the true effect sizes $\left(a_{l, B}\right)$ differ. The covariance of the polygenic scores with true additive genetic values is then

$$
\operatorname{Cov}(P, G)=\sum_{l} a_{l, A} a_{l, B} \operatorname{Var}\left(g_{l}\right) .
$$

Therefore the correlation between polygenic scores and additive genetic values is

$$
\begin{aligned}
\operatorname{Corr}(P, G) & =\frac{\sum_{l} a_{l, A} a_{l, B} \operatorname{Var}\left(g_{l}\right)}{\sqrt{\operatorname{Var}(P) \operatorname{Var}(G)}}, \text { where } \\
\operatorname{Var}(P) & =\sum_{l=1}^{L_{s}} a_{l, A}^{2} \operatorname{Var}\left(g_{l}\right), \text { and } \\
\operatorname{Var}(G) & =\sum_{l=1}^{L_{s}+L_{r}} a_{l, B}^{2} \operatorname{Var}\left(g_{l}\right) .
\end{aligned}
$$

For a neutrally evolving trait, effect sizes and heterozygosities are independent and so

$$
\operatorname{Corr}(P, G)=\operatorname{Corr}\left(a_{l, A}, a_{l, B}\right) \frac{\sum_{l} \operatorname{Var}\left(g_{l}\right)}{\sqrt{\left(\sum_{l=1}^{L_{s}} \operatorname{Var}\left(g_{l}\right)\right)\left(\sum_{l=1}^{L_{s}+L_{r}} \operatorname{Var}\left(g_{l}\right)\right)}}
$$

such that the neutral drop in prediction accuracy due to GxE is simply the decrease from 1 of the correlation of effect sizes between A and B.

\section{A.3 Modeling Details}

\section{A.3.1 Background}

Assuming Gaussian stabilizing selection and that the population mean stays close to its optimum value, selection at an individual locus is well described by a model of underdominance where the per-generation change in an allele's frequency $x$ can be described by its mean and variance,

$$
\begin{aligned}
\mathrm{E}(\Delta x) & \approx s x(1-x)(x-1 / 2) \\
\operatorname{Var}(\Delta x) & \approx \frac{x(1-x)}{2 N},
\end{aligned}
$$

where $s=\frac{a^{2}}{w^{2}}$ is the the selection coefficient, with $a$ being the additive effect of the allele with frequency $x$ relative to the alternate allele, and $N$ is the diploid population size. This approximation further assumes that the phenotypic variance is much smaller than the width of the stabilizing selection $\left(\sigma^{2} \ll w\right)$.

Assuming an infinite sites model, the mean time that a mutation spends in a certain frequency interval before fixation or loss (at a single site) multiplied by its population mutation rate is equivalent to the expected number of alleles segregating at those frequencies at a single time point (see Sawyer and Hartl, 1992). The mutation rate of an allele with effect $a$ is $2 N U \operatorname{Pr}(a)$, where $U$ is the per-generation mutation rate for the entire mutational target, and $\operatorname{Pr}(a)$ is the proportion of mutations with effect $a$ (with density $f\left(a \mid \sigma_{a}\right)$, where $\sigma_{a}$ is the standard deviation of the mutant effect size distribution). The mean time $\tau$ spent in frequency interval $(x, x+d x)$ for a new mutation with effect $a$ can be solved using the Green's function (see equation A18 of Simons et al., 2018), such that

$$
\tau(x \mid a, w)=\frac{\sqrt{\frac{N \pi}{2 s}} \exp \left(2 N s\left(x-\frac{1}{2}\right)^{2}\right)(\operatorname{erf}(\gamma)+\operatorname{erf}(\gamma(1-2 x)))\left(\operatorname{erf}(\gamma)-\operatorname{erf}\left(\gamma\left(1-\frac{1}{N}\right)\right)\right)}{\operatorname{erf}(\gamma) x(1-x)}
$$


where erf is the error function and $\gamma=\sqrt{\frac{N s}{2}}$. Thus we can solve for the expected total additive genic variance at equilibrium by summing over the heterozygosities contributed by all sites,

$$
\mathrm{E}\left(V_{a} \mid w, \sigma_{a}\right) \approx 2 N U \int_{-\infty}^{\infty} \int_{1 / 2 N}^{1-1 / 2 N} 2 a^{2} x(1-x) \tau(x \mid a, w) f\left(a \mid \sigma_{a}\right) d x d a,
$$

\section{A.3.2 Results}

In our models we focus on divergence without gene flow between a pair of populations, in which case they only share polymorphisms that arose in their common ancestor and were not lost in either population. To predict the loss of ancestral heterozygosity and probability that the descendant populations share polymorphisms, and thus describe the proportion of additive genic variance in one population explained by polymorphisms identified by a GWAS in the other, we consider the frequency trajectory of ancestrally segregating variants. To fully calculate these quantities we would need the diffusion transition density with underdominant selection to calculate the distribution of the frequency in the current day given the ancestral frequency, but we can build simple approximations of the combined effects of selection and drift. If we assume that the frequency of our allele $x$ is close to 0 such that $1-x \approx 1$, then we can approximate its expected per-generation change (eqn (A.14)) as

$$
\mathrm{E}(\Delta x) \approx s x(x-1 / 2),
$$

such that its deterministic frequency trajectory follows the logistic function from starting frequency $x_{0}$,

$$
\mathrm{E}\left(x_{t} \mid a, w\right) \approx \frac{1 / 2}{1-\left(\exp (s t / 2) \times\left(1-1 / 2 x_{0}\right)\right)}, \text { where } s=\frac{a^{2}}{w^{2}}
$$

To consider the effects of genetic drift on the frequency trajectory, we use the beta approximation to genetic drift provided by the Balding-Nichols model (Balding and Nichols, 1994). We assume that the effects of drift can be accommodated by allowing the frequency to be beta-distributed around its deterministic frequency trajectory as follows,

$$
\begin{aligned}
g\left(x_{t} \mid x_{0}, a, w\right) & =\frac{x_{t}^{\alpha-1}\left(1-x_{t}\right)^{\beta-1}}{B(\alpha, \beta)}, \text { where } \\
\alpha & =\frac{1-F}{F} \mathrm{E}\left[x_{t} \mid a\right]=(2 N / t-1) \mathrm{E}\left[x_{t} \mid a\right], \\
\beta & =\frac{1-F}{F}\left(1-\mathrm{E}\left[x_{t} \mid a\right]\right)=(2 N / t-1)\left(1-\mathrm{E}\left[x_{t} \mid a\right]\right) .
\end{aligned}
$$

Reduction in variance due to drift and selection We can predict the additive genic variance contributed by all ancestral polymorphisms (anc) to the descendent (desc) after $t$ generations by averaging over their effect sizes, starting frequencies, and trajectories,

$\mathrm{E}\left(V_{a[\text { anc, desc }]}\left(a, t, w, \sigma_{a}\right)\right)=2 N U \int_{-\infty}^{\infty} \int_{1 / 2 N}^{1-1 / 2 N} \int_{0}^{1} 2 a^{2} x_{t}\left(1-x_{t}\right) g\left(x_{t} \mid x_{0}, a, w\right) \tau\left(x_{0} \mid a, w\right) f\left(a \mid \sigma_{a}\right) d x_{t} d x_{0} d a$.

See Figure S2 for a version of Figure 1 using this approximation.

Variance in ancestor explained by polymorphisms in descendant To understand the ancestral variance attributable to present-day polymorphisms, we need to think of the properties of present-day alleles backward in time. For alleles with effect sizes large enough for selection to act strongly, selection (usually) constrains them from reaching appreciable frequencies and selection acts approximately like additive selection against the allele (eqn (A.17)). Conditional on the present-day frequency, the distribution of a deleterious, additive allele's trajectory backward in time is the same as the process forward in time, such that $g\left(x_{t} \mid x_{0}, a, w\right)=g\left(x_{0} \mid x_{t}, a, w\right)$ (Maruyama and Kimura, 1975). Therefore, we can write the expected 
additive genic variance in the ancestral population explained by variation segregating in the descendant population, in which they have diverged for $t$ generations, as

$$
\mathrm{E}\left(V_{a[\mathrm{desc}, \operatorname{anc}]}\left(a, t, w, \sigma_{a}\right)\right)=2 N U \int_{-\infty}^{\infty} \int_{1 / 2 N}^{1-1 / 2 N} \int_{0}^{1} 2 a^{2} x_{0}\left(1-x_{0}\right) g\left(x_{0} \mid x_{t}, a, w\right) \tau\left(x_{t} \mid a\right) f\left(a \mid \sigma_{a}\right) d x_{0} d x_{t} d a,
$$

which is identical to the additive genic variance contributed by all ancestral polymorphisms to the descendent (with just a flip in the subscripts, see equation (A.20)). Thus the decay in the additive genic variance segregating in an ancestral population to the present day is the same as loss in prediction accuracy when using present day polymorphisms to predict phenotypes in the ancestral population. 

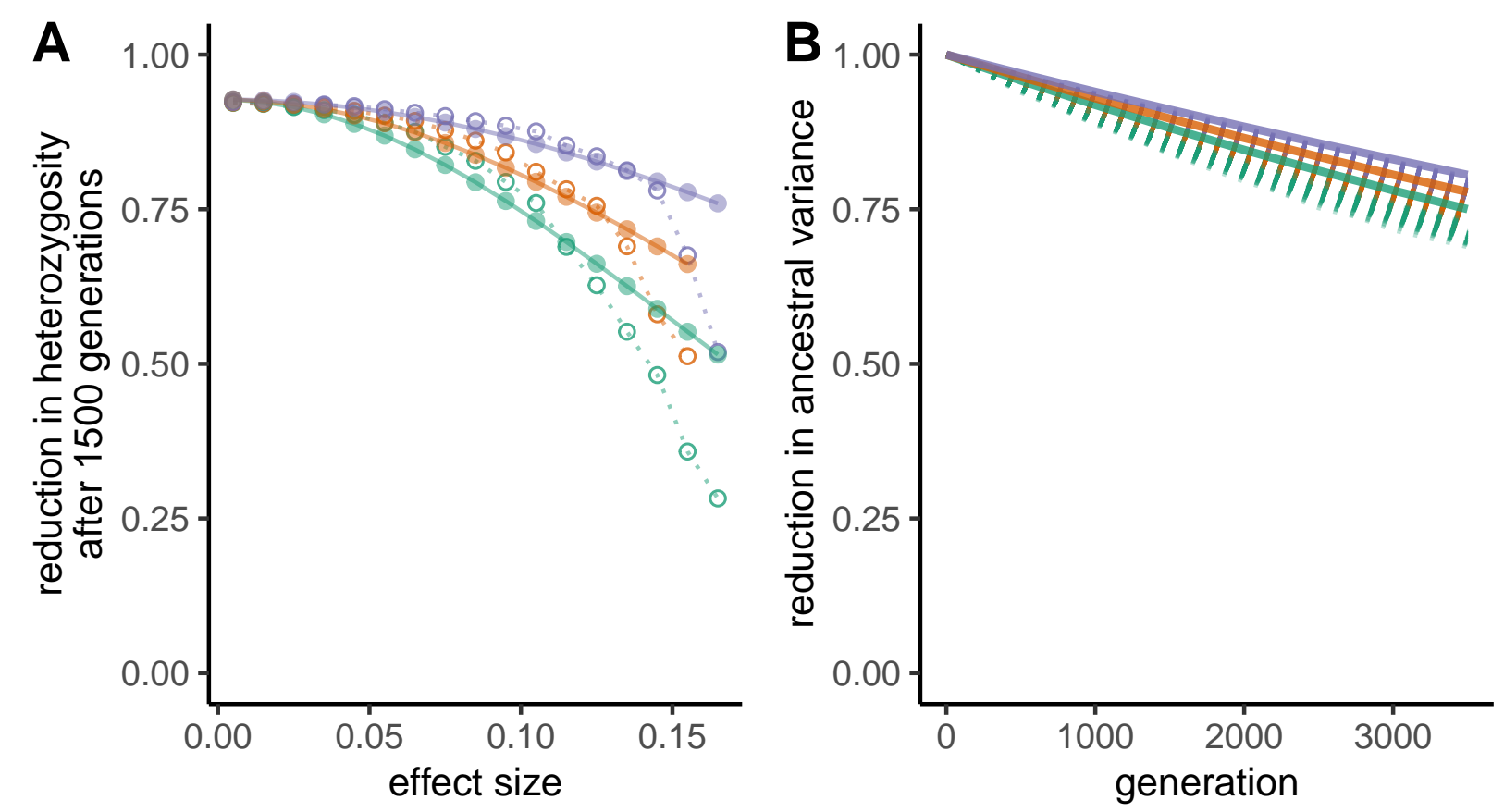

width of fitness peak

○ $w=4$ - $w=5$ - $w=7$

Figure S1: Reduction in variance due to drift and selection when the standard deviation of the mutant effect size distribution is half of that used in results presented in the main text (here $\sigma_{a}=0.05$ ). A) Reduction in heterozygosity at loci that contributed to the variance 1500 generations ago. Each open point, connected by a dashed line, represents the midpoint of the effect size bin of width 0.01 within which we averaged heterozygosity from 100 simulations. Each filled point, connected by a solid line, represents our analytical predictions for that midpoint. B) Reduction over time in the total variance contributed by polymorphisms in an ancestral population. Dashed lines show results from simulations and solid lines show analytical predictions. 

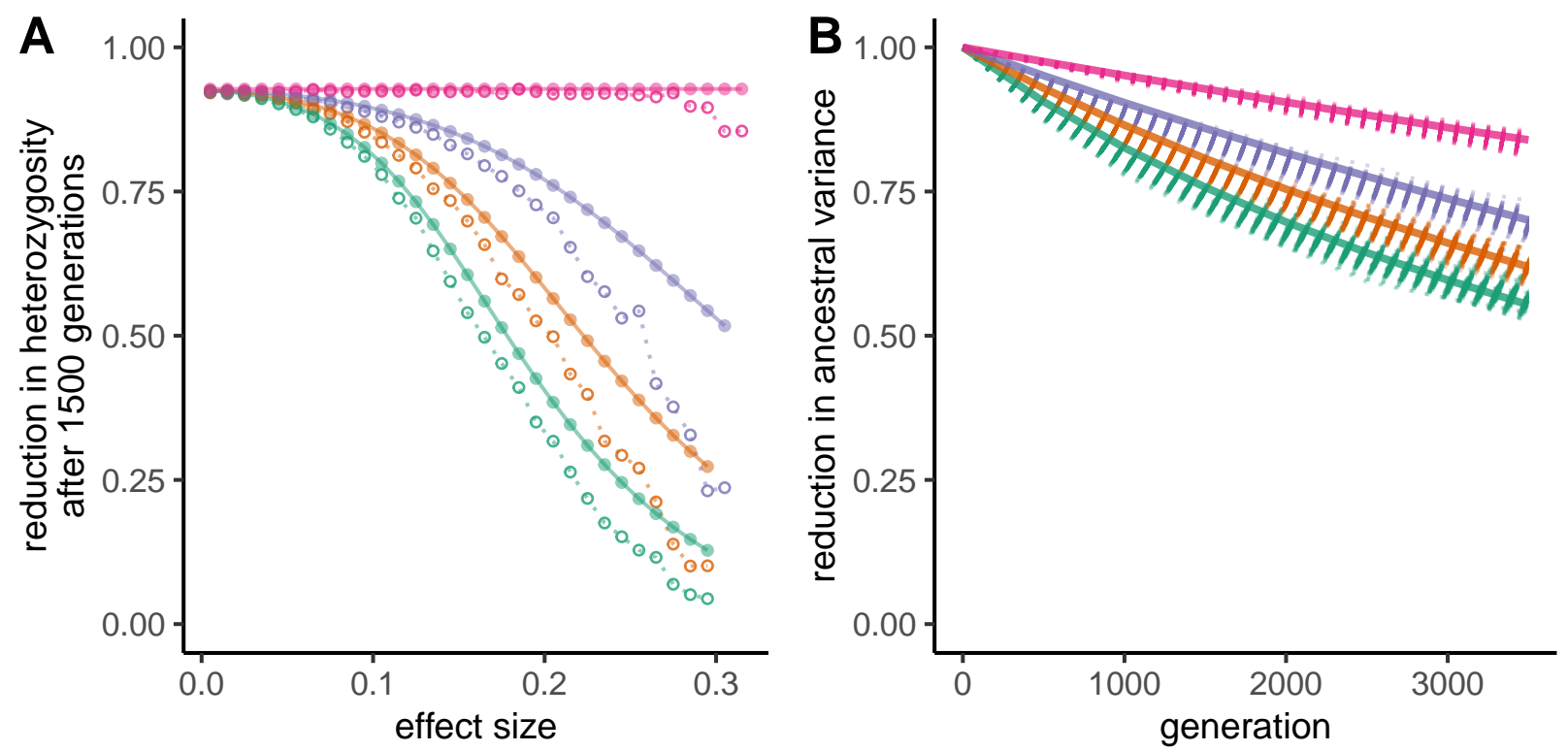

width of fitness peak

$\because \mathrm{w}=4 \rightarrow \mathrm{w}=5 \rightarrow \mathrm{w}=7 \rightarrow$ neutral

Figure S2: Reduction in variance due to drift and selection, here using analytical predictions described in Appendix A.3.2. A) Reduction in heterozygosity at loci that contributed to the variance 1500 generations ago. Each open point, connected by a dashed line, represents the midpoint of the effect size bin of width 0.01 within which we averaged heterozygosity from 100 simulations. Each filled point, connected by a solid line, represents our analytical predictions for that midpoint. B) Reduction over time in the total variance contributed by polymorphisms in an ancestral population. Dashed lines show results from simulations and solid lines show analytical predictions. 

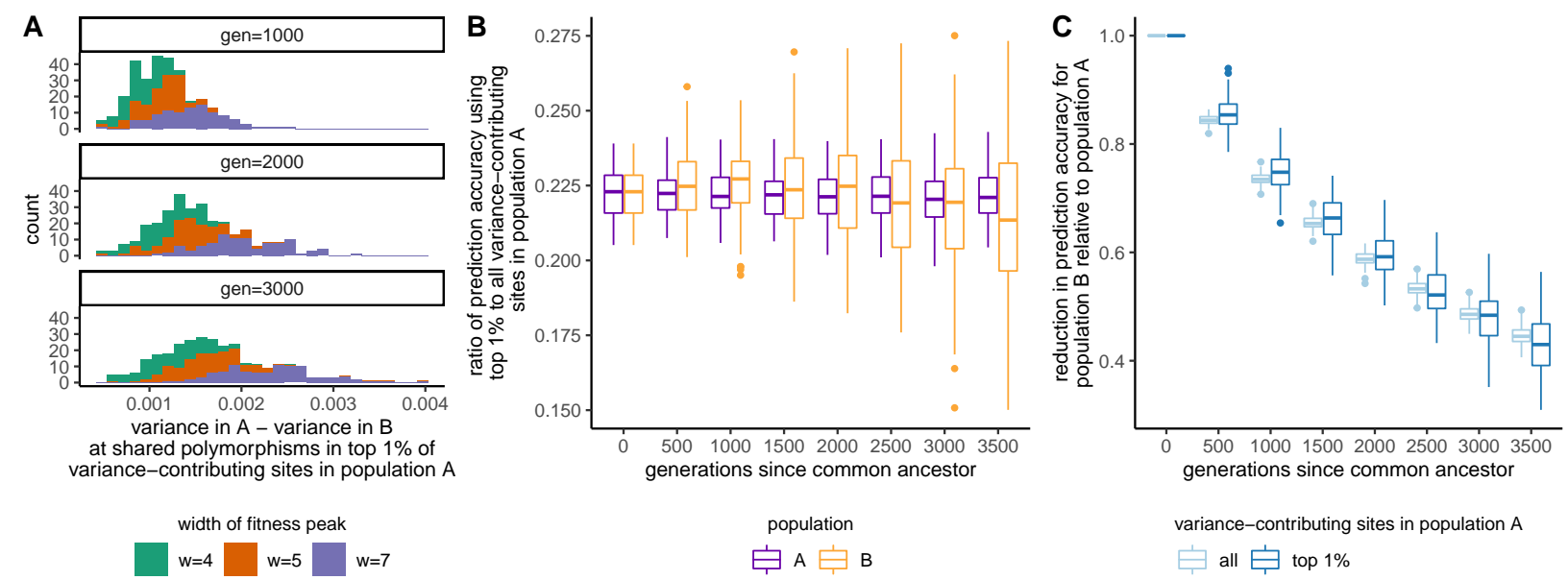

Figure S3: A) Histograms of observed differences in the variance contributed by populations A and B for shared polymorphisms in the top $1 \%$ of variance-contributing sites in population A. All differences are positive, referring to the polymorphism contributing greater variance in population A than population B. These results are consistent across all generations of divergence that we investigated, we simply show three of those here. B) For a particular population, generation, and simulation, we take the ratio of the reduction in prediction accuracy when ascertaining the top $1 \%$ of variance-contributing polymorphisms in population $\mathrm{A}$ to the reduction when ascertaining all variance-contributing polymorphisms in population A. The boxplots show the distribution of these ratios for each population and generation since the common ancestor of A and B. C) For a particular simulation, generation, and set of ascertained sites, we take the ratio of the reduction in prediction accuracy for population $\mathrm{B}$ to the reduction in population $\mathrm{A}$. The boxplots show the distribution of these ratios for each set of ascertained sites and generation since the common ancestor of A and B. 
bioRxiv preprint doi: https://doi.org/10.1101/2021.09.10.459833; this version posted September 11, 2021. The copyright holder for this preprint (which was not certified by peer review) is the author/funder, who has granted bioRxiv a license to display the preprint in perpetuity. It is made available under aCC-BY 4.0 International license.

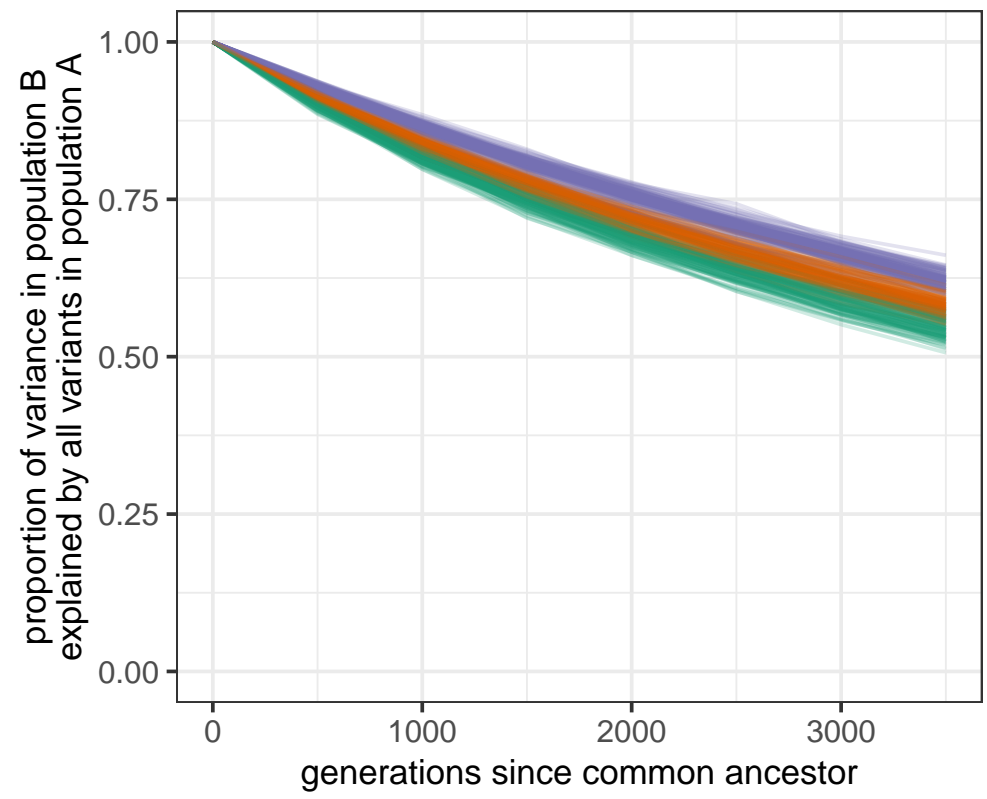

width of fitness peak

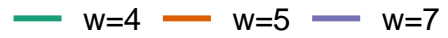

Figure S4: Prediction accuracy in population B when ascertaining all polymorphisms in population A, using half of the standard deviation of the mutant effect size distribution as results in the main text (here $\left.\sigma_{a}=0.05\right)$. Lines show results from simulations. 

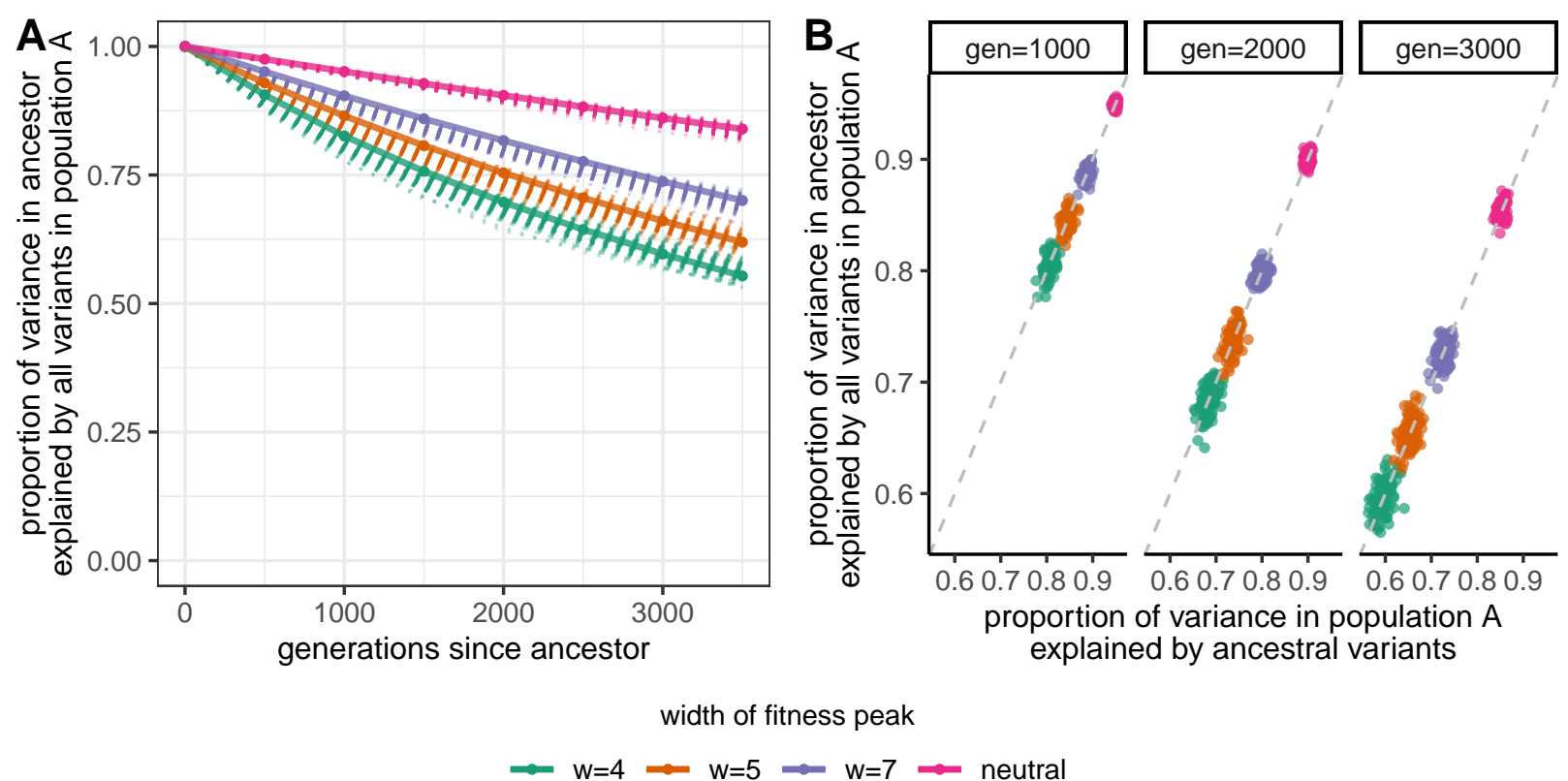

Figure S5: A) Reduction in prediction accuracy in population ancestral to population A with increasing divergence time between them. Dashed lines show results from simulations and solid lines show analytical predictions. B) At particular time depths of divergence, the reduction in prediction accuracy in ancestral population (y-axis) is about the same as reduction in ancestral variance (x-axis). Points represent results from a single simulation. Dashed line is line of equality. 


\section{Directional Selection}

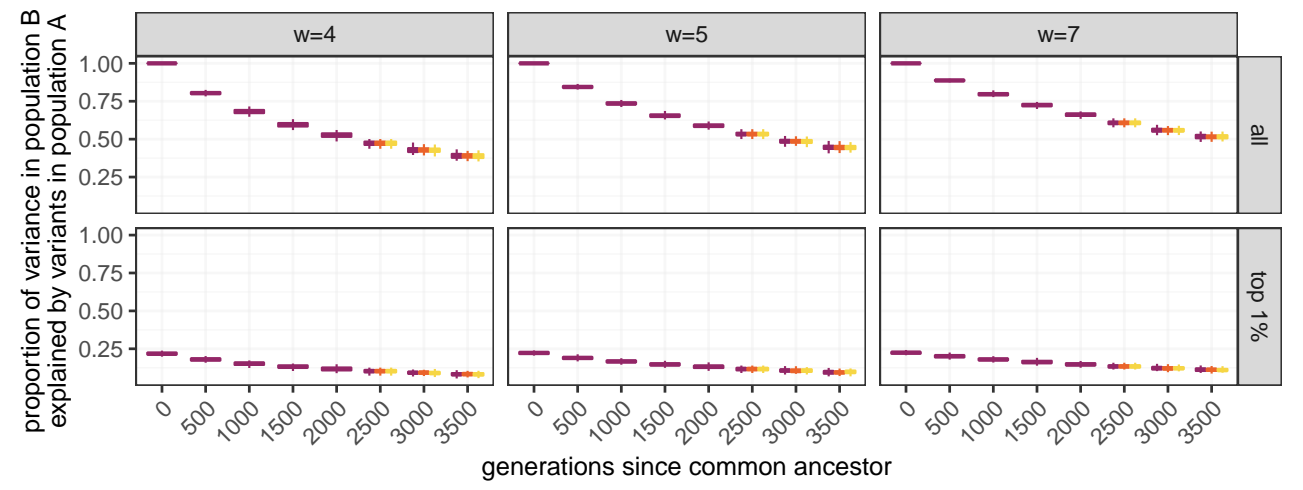

optimum shift (\# of standard deviations)

追 0 兒 1 2

GxE

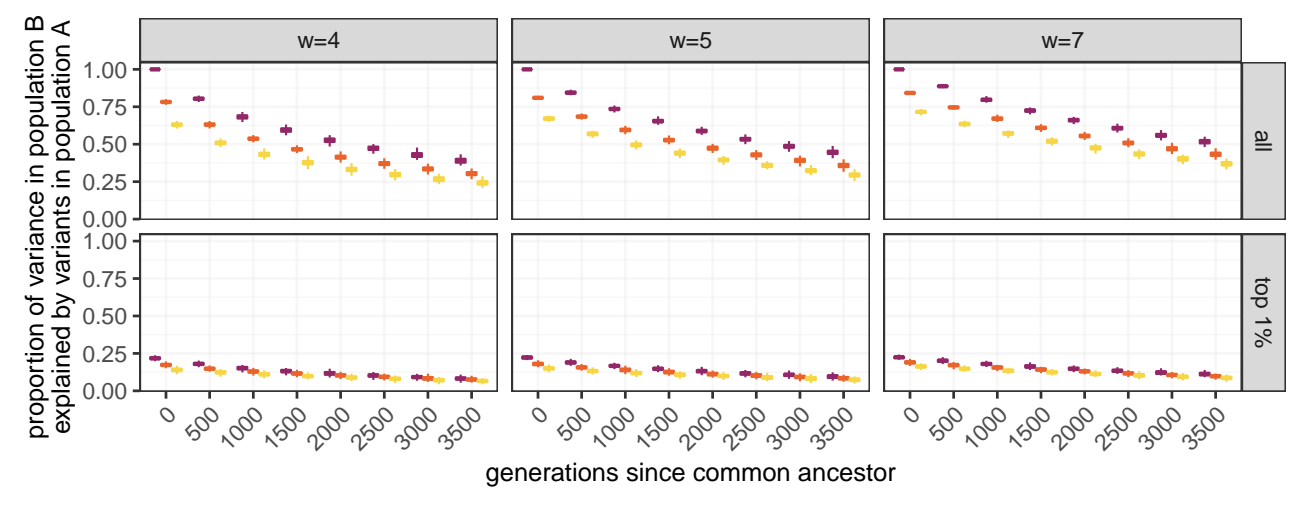

effect size correlation among populations

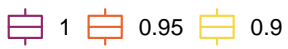

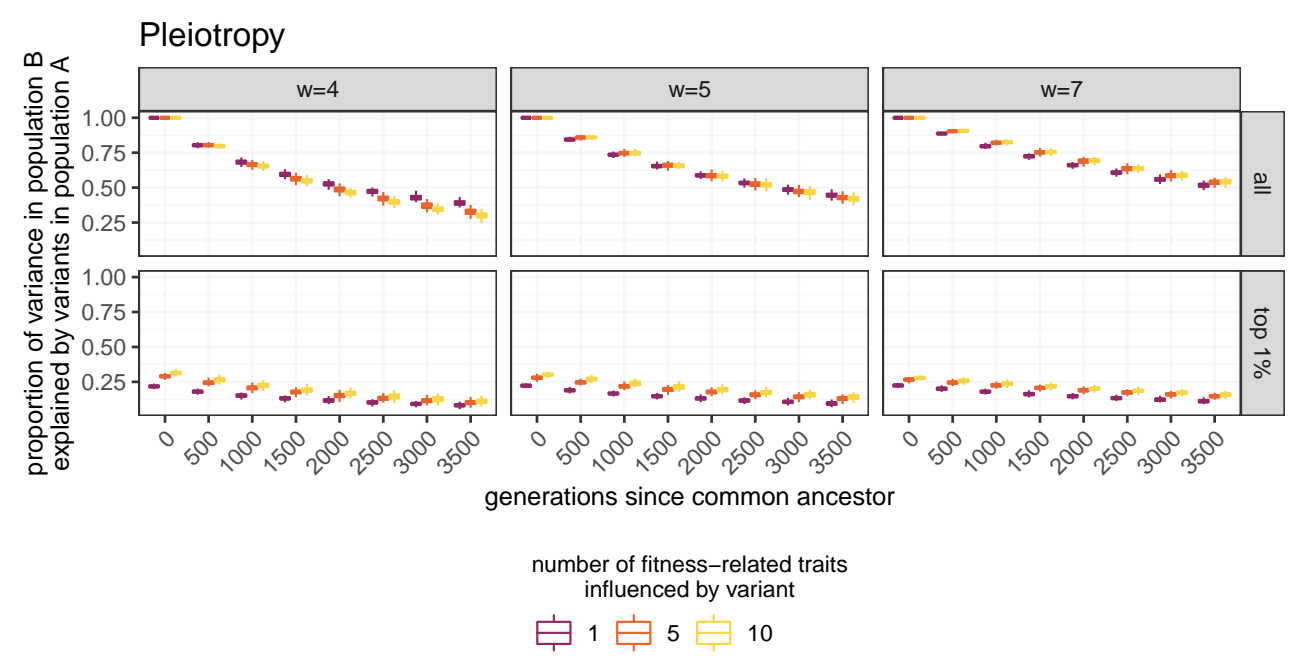

Figure S6: Comparison of the baseline stabilizing scenario and each extension (labelled by title) for the reduction in prediction accuracy in population B. Boxplots show the distribution of observations from 100 simulations, and are colored by the parameter values that vary in a particular extension. Results from the baseline scenario are always represented in purple (the first parameter value in each legend). Each column represents a different width of the fitness peak, and each row represents the set of polymorphisms ascertained in population A (top: all, bottom: top $1 \%$ based on variance contributed). 

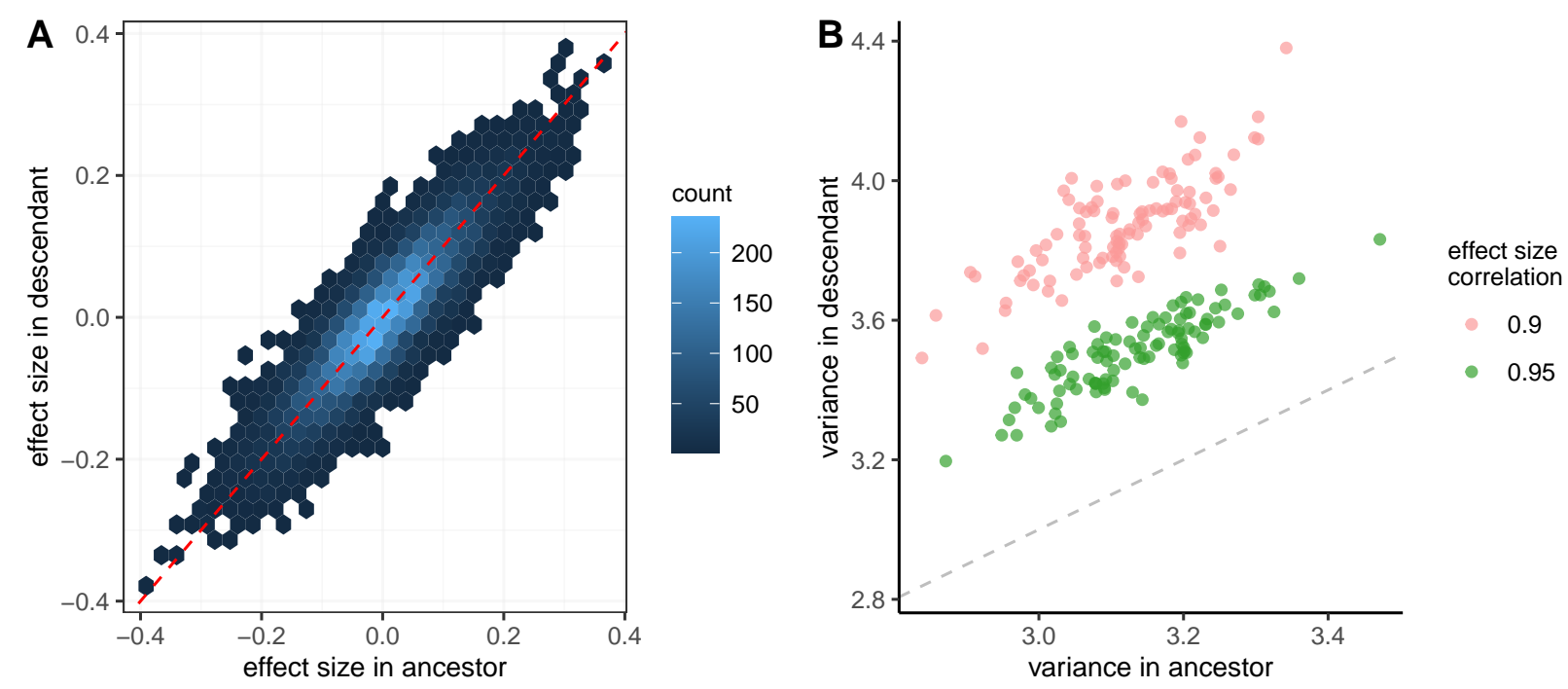

Figure S7: Shift of effect sizes and within-population variances when there's GxE and populations A and B begin to experience different environments from their common ancestor at the time of divergence. A) Changes in effect sizes from the ancestral population (x-axis) to one of its descendant populations (y-axis) for each variance-contributing polymorphism. Colors represent the number of sites in a particular bin (outlined by hexagon). Results are shown for a single simulation with $w=5$ and effect size correlation of 0.9 . B) At the time of divergence/environmental shift, the variance in the descendant population (y-axis) increases relative to the variance in the ancestral population (x-axis) due to the shift in effect sizes. Each point represents a single simulation. Dashed lines in A and B represent lines of equality. 


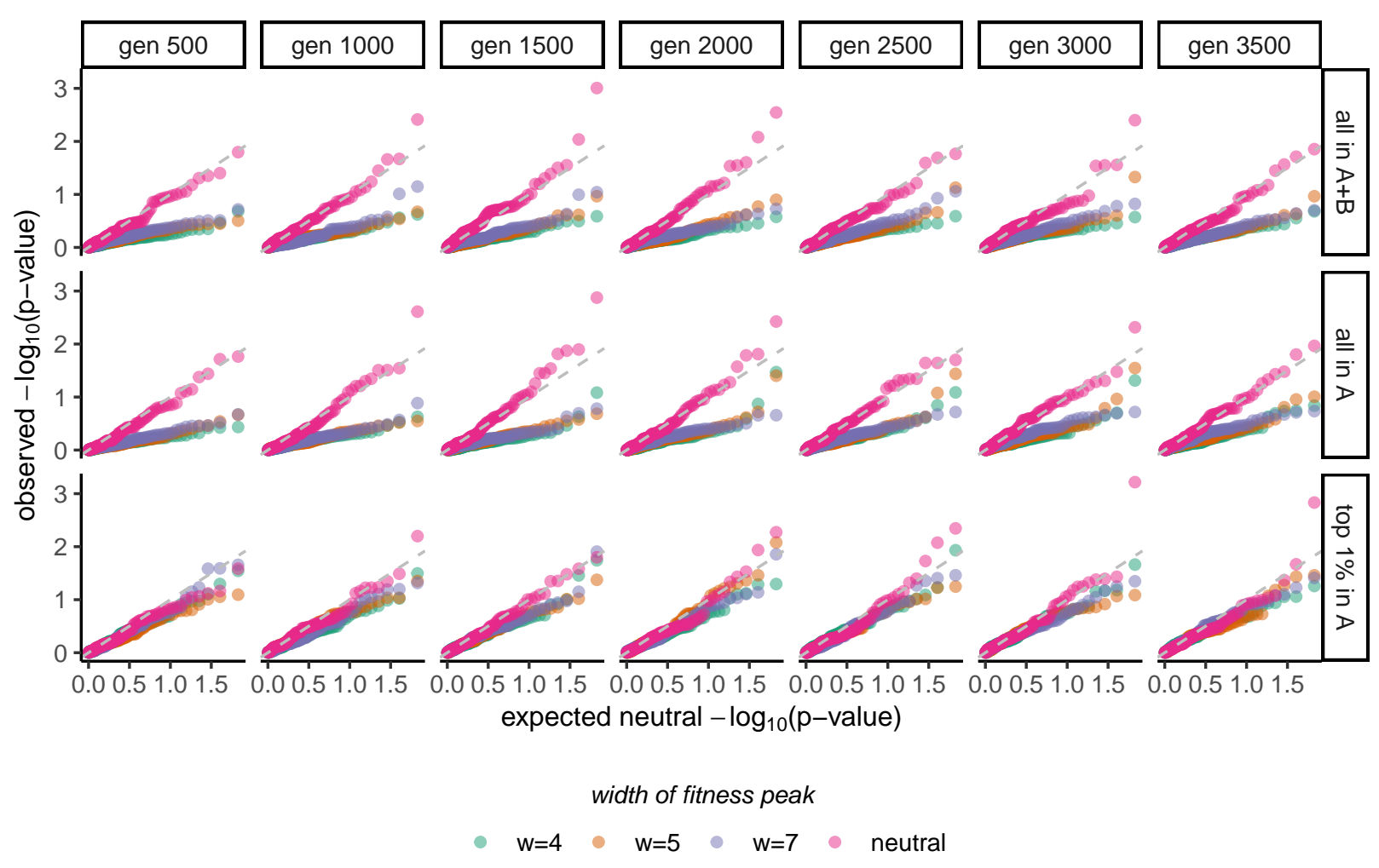

Figure S8: Quantile-quantile plot of observed p-values for $Q_{X}$ under the baseline case against expected pvalues under neutrality (uniform distribution). The dashed line shows equality; points that lie above this line indicate that the observed distribution has a higher density of low p-values than the neutral distribution, and points that lie below it indicate the opposite. Columns show results from every 500 generations of divergence between populations $\mathrm{A}$ and $\mathrm{B}$ and rows show results from different sets of ascertained polymorphims (top: all polymorphisms from both populations, middle: all polymorphisms in A, bottom: top $1 \%$ of variancecontributing polymorphisms in A). 


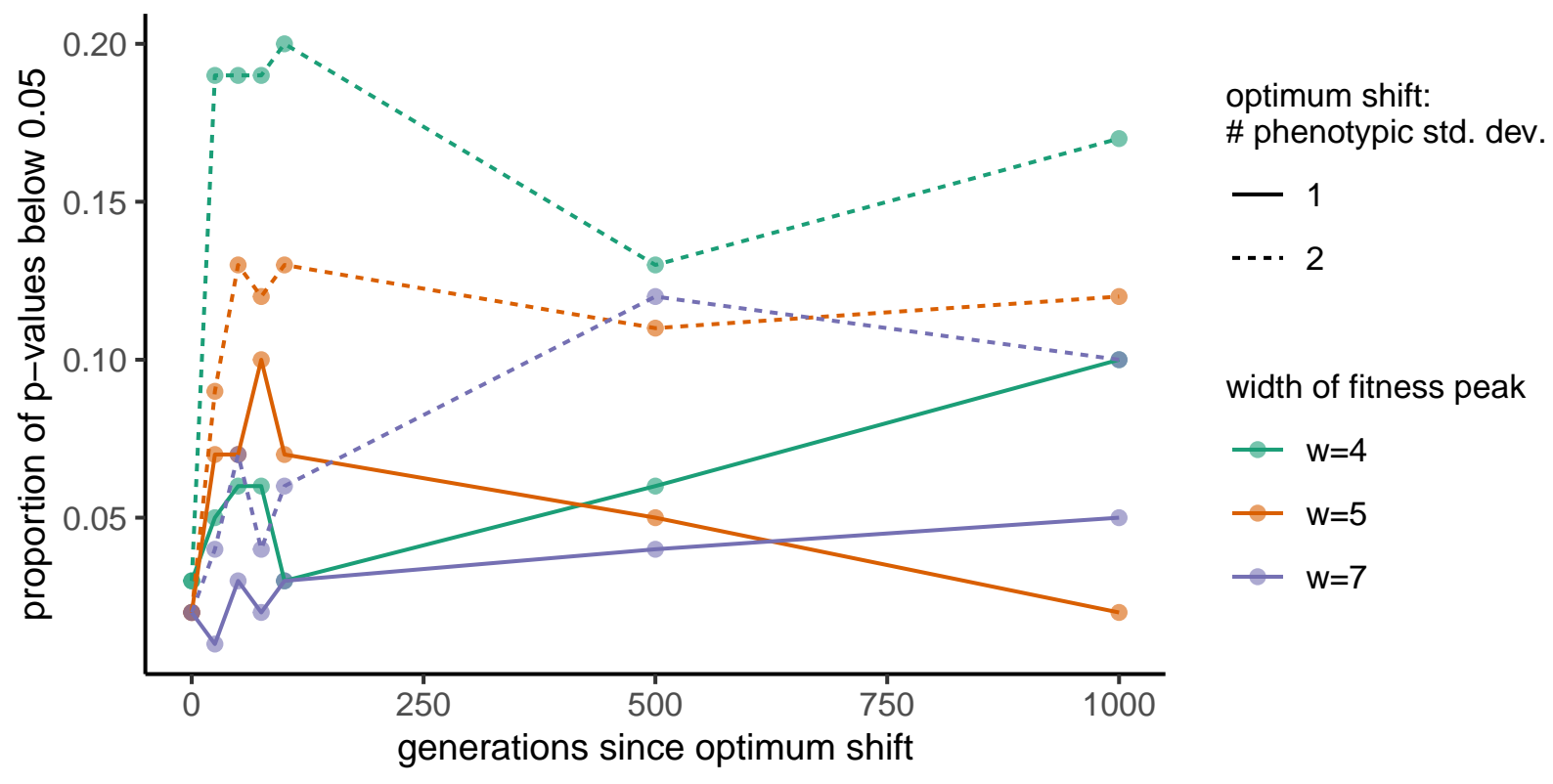

Figure S9: Under the directional selection scenario, proportion of p-values below the significance threshold of 0.05 with time since the shift in the optimum (the shift began 2500 generations of divergence between populations A and B). Each point is averaged across 100 simulations. Solid lines connect points corresponding to an optimum shift of one standard deviation of the phenotypic distribution, while dashed lines connect points corresponding to an optimum shift of two standard deviations. Following the optimum shift, we recorded results every 25 generations for the first 100 generations, and then every 500 generations.

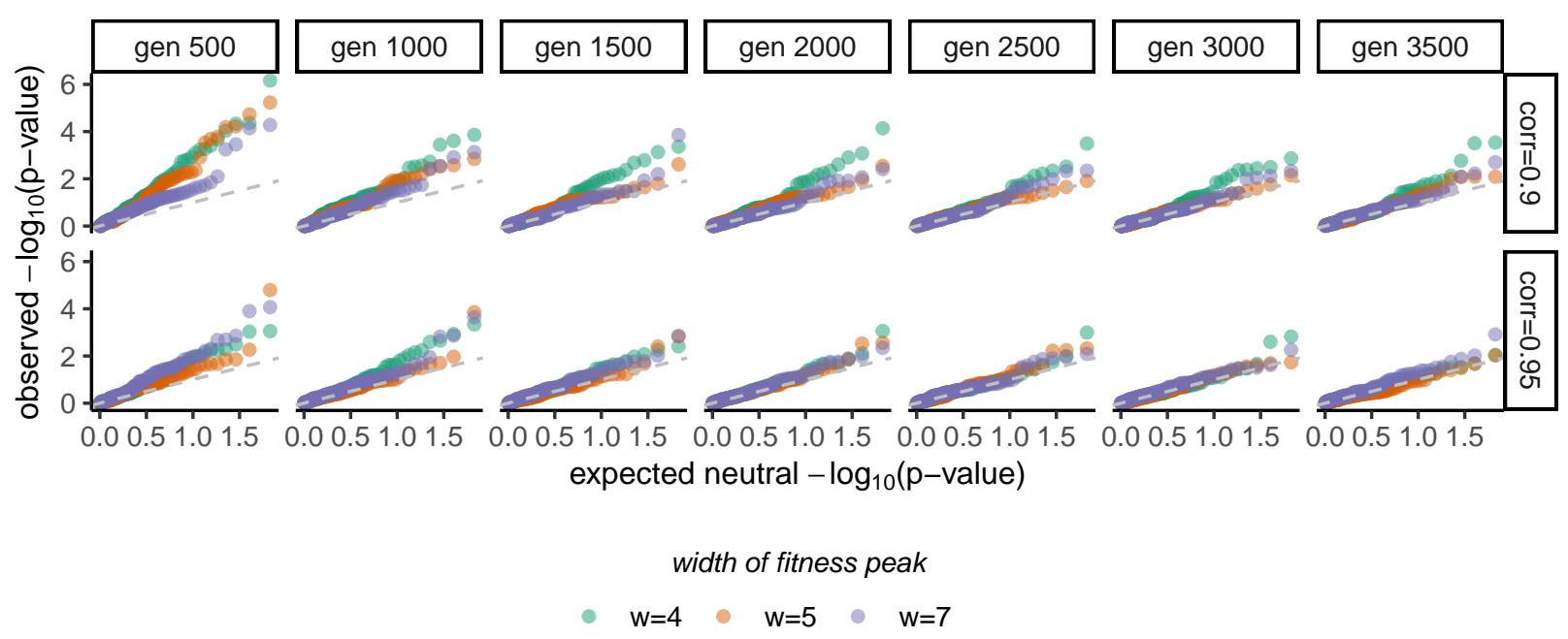

Figure S10: Quantile-quantile plot of observed p-values for $Q_{X}$ under $\mathbf{G x E}$ against expected p-values under neutrality (uniform distribution). The dashed line shows equality; points that lie above this line indicate that the observed distribution has a higher density of low p-values than the neutral distribution, and points that lie below it indicate the opposite. Columns show results from every 500 generations of divergence between populations A and B and rows show results from each simulated correlation of effect sizes among populations. 


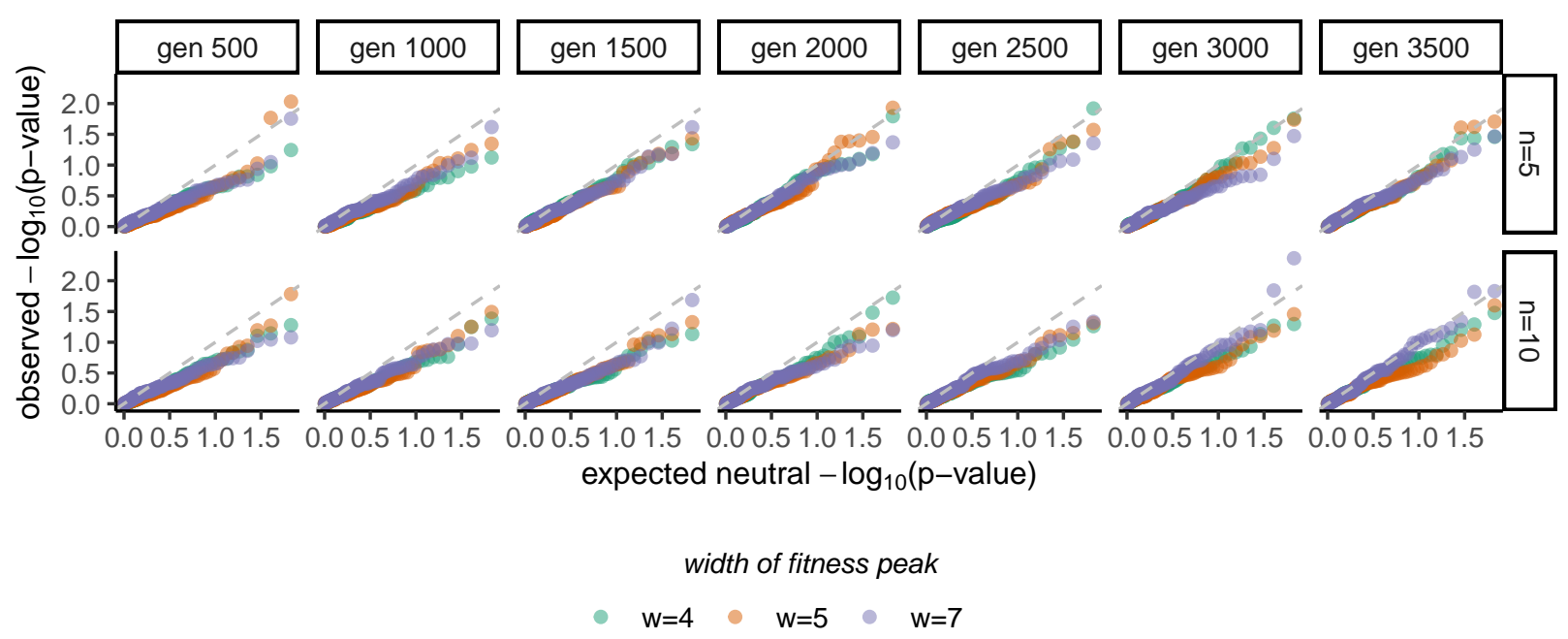

Figure S11: Quantile-quantile plot of observed p-values for $Q_{X}$ under pleiotropy against expected p-values under neutrality (uniform distribution). The dashed line shows equality; points that lie above this line indicate that the observed distribution has a higher density of low p-values than the neutral distribution, and points that lie below it indicate the opposite. Columns show results from every 500 generations of divergence between populations $\mathrm{A}$ and $\mathrm{B}$ and rows show results from each simulated number of fitness-related traits $(n)$ influenced by a mutation. 

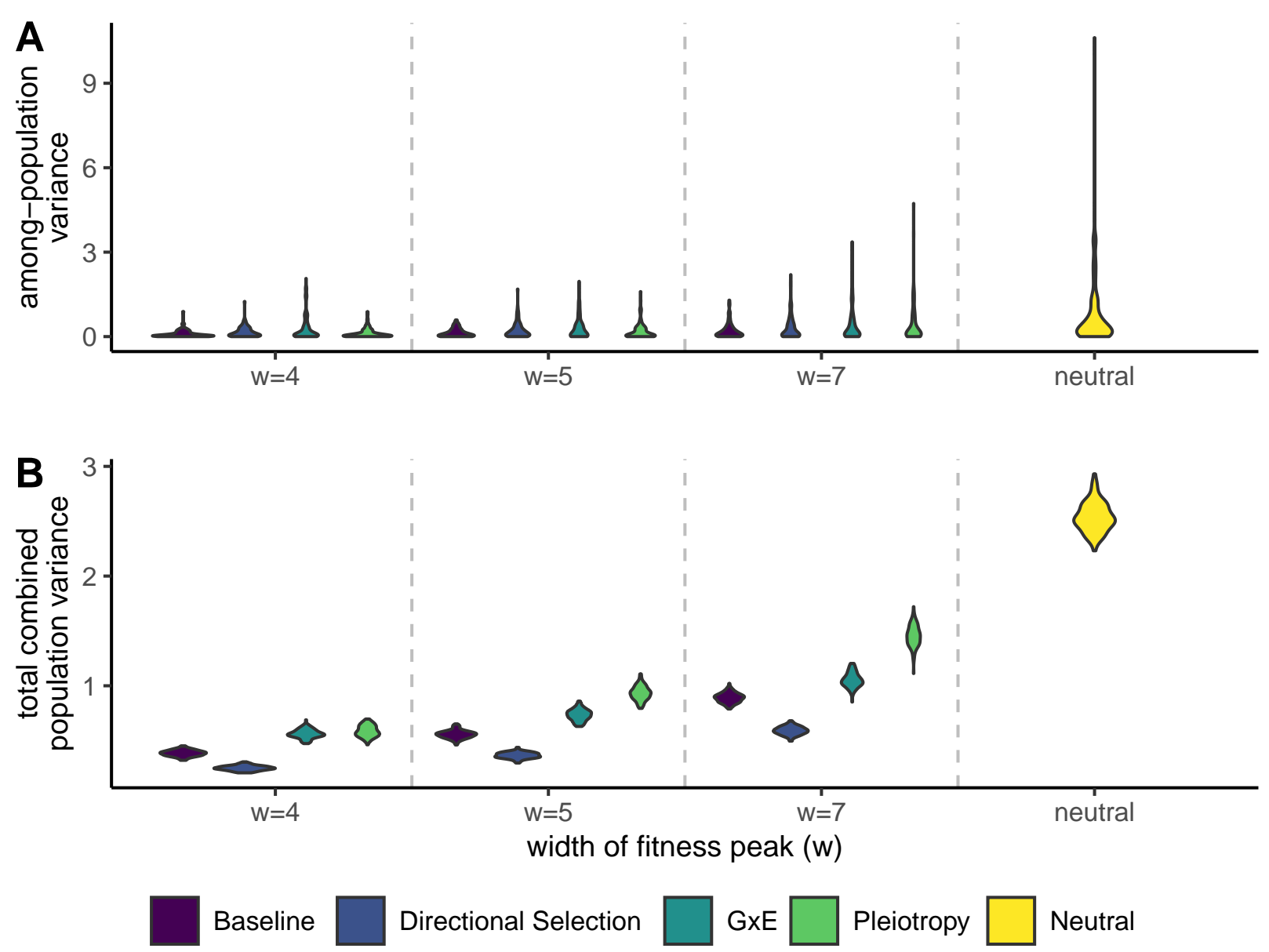

Figure S12: Components of $Q_{S T}$ when estimated from the top $1 \%$ of variance-contributing sites in population A. Results for GxE are shown for the effect size correlation 0.9 and results for directional selection are shown for an optimum shift of two phenotypic standard deviations. A) The estimated mean difference in polygenic scores. The larger this value the larger the among-population variance, which is the numerator of $Q_{S T}$. B) The total variance of the populations combined, which is the denominator of $Q_{S T}$. 